\title{
Hierarchical Notification Dissemination for IMS Presence using Network Coordinates
}

by

Dylan Andrew Harper Smith, B. Eng.

A thesis submitted to the Faculty of Graduate and Postdoctoral Affairs

in partial fulfillment of the requirements for the degree of

Master of Applied Science in Electrical and Computer Engineering

Ottawa-Carleton Institute for Electrical and Computer Engineering (OCIECE)

Department of Systems and Computer Engineering

Carleton University

Ottawa, Ontario, Canada, K1S 5B6

December, 2010

(C) Copyright 2010, Dylan Andrew Harper Smith 
Library and Archives

Canada

Published Heritage

Branch

395 Wellington Street Ottawa ON K1A ON4 Canada
Bibliothèque et

Archives Canada

Direction du

Patrimoine de l'édition

395 , rue Wellington

Ottawa ON K1A ON4

Canada
Your file Votre référence

ISBN: 978-0-494-79556-9

Our file Notre référence

ISBN: 978-0-494-79556-9
NOTICE:

The author has granted a nonexclusive license allowing Library and Archives Canada to reproduce, publish, archive, preserve, conserve, communicate to the public by telecommunication or on the Internet, loan, distribute and sell theses worldwide, for commercial or noncommercial purposes, in microform, paper, electronic and/or any other formats.

The author retains copyright ownership and moral rights in this thesis. Neither the thesis nor substantial extracts from it may be printed or otherwise reproduced without the author's permission.
AVIS:

L'auteur a accordé une licence non exclusive permettant à la Bibliothèque et Archives Canada de reproduire, publier, archiver, sauvegarder, conserver, transmettre au public par télécommunication ou par l'Internet, prêter, distribuer et vendre des thèses partout dans le monde, à des fins commerciales ou autres, sur support microforme, papier, électronique et/ou autres formats.

L'auteur conserve la propriété du droit d'auteur et des droits moraux qui protège cette thèse. Ni la thèse ni des extraits substantiels de celle-ci ne doivent être imprimés ou autrement reproduits sans son autorisation.
In compliance with the Canadian Privacy Act some supporting forms may have been removed from this thesis.

While these forms may be included in the document page count, their removal does not represent any loss of content from the thesis.
Conformément à la loi canadienne sur la protection de la vie privée, quelques formulaires secondaires ont été enlevés de cette thèse.

Bien que ces formulaires aient inclus dans la pagination, il n'y aura aucun contenu manquant. 


\section{Abstract}

A presence service provides event-based notifications of the status of contacts. Currently duplicate notifications must be sent directly to a set of watchers in unicast messages, putting unneeded strain on the outgoing network links of the source node. We propose an extension to the Session Initiation Protocol (SIP) to distribute notifications through a tree of watchers using network coordinates to build the tree.

Simulations were performed using variations of the input parameters to test the scalability, reliability, notification delay, and load on the network under different conditions. The results show traffic costs halved by using the extension without significantly adding delay, even as additional users are added to the simulation with the same set of server nodes. The simulation showed the packet loss and node failure could be handled, but high rates of these network faults would increase notification delay and the load on the network. 


\section{Acknowledgments}

I appreciate the financial support from the NSERC grant \#CRDPJ 354729-07 and the OCE grant \#CA-ST-150764-8. 


\section{Table of Contents}

Abstract

Acknowledgments $\quad$ iv

Table of Contents $\quad$ v

List of Tables viii

List of Algorithms $\quad$ ix

List of Figures $\quad$ x

Abbreviations $\quad$ xii

Terminology xiv

1 Introduction 1

1.1 Motivation and objectives .................. 2

1.2 Solution and Contributions . . . . . . . . . . . . . . 3

1.3 List of publications . . . . . . . . . . . . . . . . . 4 
2 Background 5

2.1 IP Multimedia Subsystem . . . . . . . . . . . . . 7

2.2 Session Initiation Protocol . . . . . . . . . . . . . 11

2.3 Presence Server Migration . . . . . . . . . . . . . . . 13

2.4 Presence Data . . . . . . . . . . . . . . . . . . 15

2.5 Network Coordinates . . . . . . . . . . . . . . . 16

3 State of the Art in Notification Dissemination $\quad 20$

3.1 IP Multicast . . . . . . . . . . . . . . . . . . . . 21

3.2 Aggressive Tree Optimization _. . . . . . . . . . . . . 22

3.3 Peer-to-peer ........................... 23

3.4 Minimum Spanning Tree Construction . . . . . . . . . . . . . . . 24

3.5 Location unaware tree construction . . . . . . . . . . . . 26

3.6 Temporary Tree Construction . . . . . . . . . . . . . 27

4 Proposed Solution $\quad 29$

4.1 Notification Traffic Reduction Problem . . . . . . . . . . . . 29

4.2 Proposed Solution . . . . . . . . . . . . . . . . . . 31

4.3 Building the Distribution Tree . . . . . . . . . . . . . 33

4.4 Process of Leaving the Tree . . . . . . . . . . . . . . . 37

4.5 Maintaining the Distribution Tree . . . . . . . . . . . . . . . 39

4.6 Compatibility with IMS Presence . . . . . . . . . . . . . . . 42

5 Evaluation of Proposed Solution $\quad 44$

5.1 Evaluation Criteria . . . . . . . . . . . . . . . 44

5.1.1 Traffic Cost ..................... 45 
5.1 .2 Notification Delay . . . . . . . . . . . . . . . 47

5.1 .3 Reliability ................... 50

5.1 .4 Scalability ....................... 51

5.2 Simulation Model and Implementation . . . . . . . . . . . 51

5.3 Simulation Experiment Design . . . . . . . . . . . . . . 59

5.4 Evaluation Results . . . . . . . . . . . . . . . . 61

5.4 .1 Base Scenario . . . . . . . . . . . . . 61

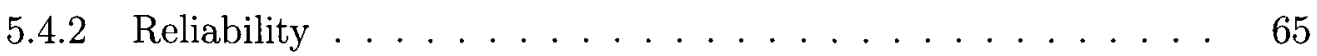

5.4 .3 Scalability . . . . . . . . . . . . . 71

6 Conclusions $\quad 73$

6.1 Summary of Contributions . . . . . . . . . . . . . 74

6.2 Future Work . . . . . . . . . . . . . . . 74

$\begin{array}{ll}\text { List of References } & 76\end{array}$

$\begin{array}{ll}\text { Appendix A Presence Information Data Elements } & 80\end{array}$

A.1 Presence Information Data Format . . . . . . . . . . . . . 80

A.2 Rich Presence Information Data Format . . . . . . . . . . . 81

A.3 Contact Information in Presence Information Data Format . . . . . . 82

A.4 Timed Presence Extension . . . . . . . . . . . . . . . 82

A.5 User Agent Capability Extension . . . . . . . . . . . . 83 


\section{List of Tables}

5.1 Subset of the matrix of millisecond delays between nodes . . . . . . 53

5.2 Average steady-state values for base scenario . . . . . . . . . . . 64 


\section{List of Algorithms}

4.1 Tree building algorithm . . . . . . . . . . . . . . 36 


\section{List of Figures}

2.1 Presence Roles $\ldots \ldots \ldots \ldots \ldots \ldots$

2.2 Resource List Server Sequence Diagram . . . . . . . . . . . . 10

3.1 Minimum Spanning Tree notification delay problem . . . . . . . . 25

3.2 Location unaware traffic problem $\ldots \ldots \ldots \ldots \ldots . \ldots . \ldots 27$

4.1 Direct Notification Traffic Problem . . . . . . . . . . . . . 30

4.2 Adding a node to the distribution tree $\ldots \ldots \ldots \ldots$

4.3 Node leaving the distribution tree $\ldots \ldots \ldots \ldots \ldots$

5.1 Transport timeline for example packets . . . . . . . . . 46

5.2 Notification Delay Example . . . . . . . . . . . . . . . . . 49

$5.3 \quad$ Star Topology . . . . . . . . . . . . . . . . . . . . . 52

5.4 average traffic cost per time interval . . . . . . . . . . 62

5.5 average notification delay per time interval $\ldots \ldots \ldots \ldots$

5.6 CDF for notification delay $\ldots \ldots \ldots \ldots \ldots \ldots$

5.7 average notification delay $\ldots \ldots \ldots \ldots \ldots \ldots$

5.8 average traffic cost $\ldots \ldots \ldots \ldots \ldots \ldots \ldots \ldots$

5.9 average notification delay $\ldots \ldots \ldots \ldots \ldots \ldots$

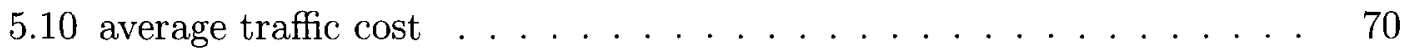

5.11 average notification delay $\ldots \ldots \ldots \ldots \ldots \ldots \ldots \ldots$ 
5.12 average traffic cost . . . . . . . . . . . 72 


\section{Abbreviations}

CDF: Cumulative Distribution Function

DNS: Domain Name Service

IMS: IP Multimedia Subsystem

IP: Internet Protocol

MST: Minimum Spanning Tree

NC: Network Coordinate

ns-2: The Network Simulator (version 2)

PIDF: Presence Information Data Format

PUA: Presence User Agent

RLS: Resource List Server

RTT: Round-trip time

SIP: Session Initiation Protocol

UDP: User Datagram Protocol 
URI: Uniform Resource Identifier

XML: Extensible Markup Language

XMPP: Extensible Messaging and Presence Protocol 


\section{Terminology}

access network: The network of an Internet service provider used to connect to the Internet.

child node: A node that receives notification through its parent node.

message: A packet that may be retransmitted.

node: Network attached device.

orphaned node: A non-root tree node without a parent.

parent node: A tree node's parent node is the node through which notifications are received.

root node: The source node for a notification tree. Does not include nodes that forward notifications in the notification tree.

source node: The primary source of direct notifications for watchers of a presentities presence status.

traffic cost: The number of packets in transport at any point in time.

transport time: The time a packet takes to transfered by the transport layer. 
watcher: A subscriber to a presentity's presence status. 


\section{Chapter 1}

\section{Introduction}

Presence is a service that provides service-subscribers updates on the status of their contacts before the subscriber attempts to initiate a multimedia session with one of them. These updates are normally sent directly to a set of destinations that have subscribed to receive these updates; however, this method causes a number of identical messages to be sent across a limited set of network links close to the notifier. Internet Protocol (IP) Multicast could reduce message replication but to date it is not widely deployed [1]. Instead, this thesis presents an application-layer multicast approach that uses a tree-based distribution of notifications, with network coordinates guiding the set up of the distribution tree.

This tree-based approach to notification distribution could be applied to any notification protocol, however, a specific protocol must be studied in order to quantitatively evaluate the presence traffic problem and solutions. The presence service as it is used in the IP Multimedia Subsystem (IMS) was used to evaluate tree-based notification distribution.

The background information on IMS, presence, and the standards on which these 
are based are explained in chapter 2 . In chapter 3 , the existing work related to reducing notification traffic is looked at in order to clearly state the remaining problems with presence traffic in section 4.1. The following sections of chapter 4 explain the proposed solution of using network coordinates to organize nodes (network attached devices) into a tree for distributing network coordinates. The solution was evaluated with an ns- 2 simulation as detailed in chapter 5 .

\subsection{Motivation and objectives}

The objective of the research described in this thesis is to increase the scalability of the presence service by reducing the network load and congestion caused by the service. Doing so would help enable the widespread deployment of the IMS architecture.

IMS is an architecture framework which aims to provide richer multimedia services for users of cellular networks by using Internet protocols, while providing the high quality of service needed for multimedia communications. This high quality of service is provided by reserving bandwidth for each multimedia session on the circuit-switched networks used by cellular networks. The convergence of these networks requires a high level of scalability, so services like presence must reduce their load on the network.

Presence traffic can be expected to increase as the number of users and the length of their contact list increase. The frequency of the presence status updates could also be expected to increase as users start relying on the presence protocol to help determine when and how to start communication with their contacts. This would be increased further by mobile devices automatically update presence information using ambient information obtained from sensors, user behaviour, and shared data 
stored on the device. Supplementary information on the presence service in IMS are provided in chapter 2.

A solution was needed that would reduce presence notification traffic without resulting in unacceptable notification delays for users. In addition, the presence notifications are not large enough to justify an approach that adds significant overhead in order to reduce the load on the network from the existing traffic. These constraints prevented existing approaches from being acceptable solutions, as discussed in chapter 3 .

\subsection{Solution and Contributions}

The proposed solution to reducing load on the network caused by presence traffic is to use a location-aware application-layer multicast. Location information is obtained from measuring the round-trip time of existing presence request-response message pairs. These measurements are then used to calculate virtual coordinates for devices providing the presence service using an existing network coordinate algorithm. These network coordinates are shared between these devices by attaching the network coordinate data to existing network packets through an extension to the event notification framework used to provide the presence service.

Subscribers to a user's presence status are logically organized into a tree. Their network coordinates are used to place nodes that are close together into the same part of the tree. The communication needed to organize nodes into a distribution tree is done by including this information with the notifications of presence status changes. This proposed protocol extension, which is discussed in detail in chapter 4, 
requires few extra messages by performing lazy tree construction and piggy-backing information on existing traffic.

The proposed protocol extension is evaluated using a network simulator to simulate the traffic for the presence protocol. The protocol extension is shown in chapter 5 to significantly reduce network traffic with only a minor overhead for the notification delay. These evaluations include the simulation of network faults, which are handled by the protocol extension.

The contributions of this research consist of:

- A low-overhead notification distribution system that significantly reduces network traffic by using shorter network paths

- A protocol extension that can be used with the presence service in IMS between nodes that support the extension

- An evaluation of tree-based notifications for IMS presence with the simulation of network faults

\subsection{List of publications}

Accepted for publication in IEEE Global Communications Conference 2010 [2]:

- Hierarchical Notification Dissemination for IMS Presence using Network Coordinates 


\section{Chapter 2}

\section{Background}

The presence service provides a feature that has existed for years in instant messaging services to display a status indicator for each contact in a contact list. The user providing presence information is called a presentity. Presence conveys to watchers (nodes viewing presence status) the presentity's ability or willingness to communicate. This can include relevant context such as their available communication capabilities, their current activity, and description of their current environment. This can allow a watcher to quickly identify who they can communicate with from their contact list before trying to initiate a conversation, or when would be a good time to communicate with a specific contact on their list.

There are a number of implementations of the presence service for different instant messaging services. Two standardized services are the Presence Event Package for the Session Initiation Protocol (SIP) [3] and the Extensible Messaging and Presence Protocol (XMPP). There are also proprietary instant messaging applications that use presence; popular ones include America Online (AOL) Instant Messenger (AIM), Yahoo! Messenger, and Windows Live Messenger [4]. 
This thesis will focus on Presence in the IP Multimedia Subsystem (IMS), which uses the Presence Event Package for SIP [3]. This is done in order to quantify notification latency and the network load for this implementation of the presence service, and in order to compare this with the results of modifying the protocol. IMS is an architecture framework, as described in section 2.1, which is developed to provide services like presence using the SIP protocol.

The purpose of this chapter is to describe details about the standard IMS presence service to help understand the problems with presence traffic that were introduced in chapter 1 and give enough background information to describe the alternative approaches to solving the problem. The alternative approaches described in chapter 3 and 4 build on the standards described in this chapter as well as the concepts of network coordinates described in section 2.5 .

Relevant aspects of the SIP protocol are explained in section 2.2, such as transactions and dialogs that are needed for reliable communication using SIP over unreliable transport layer protocols. This chapter also covers the Presence Event Package for SIP in section 2.4 along with standardized extensions that have been adopted for IMS presence. Lastly, this chapter explains how network coordinates can be used to provide location information for application-layer multicast protocols discussed in chapter 3 and 4 . 


\subsection{IP Multimedia Subsystem}

IMS is an architecture framework, developed for providing IP (Internet Protocol) multimedia services over next generation networks. These networks would be a convergence of cellular networks and the Internet upon which services could be used through the IMS framework. These services include, but are not limited to, Voice Over IP (VoIP), videoconferencing, instant messaging, and presence [5]. These services could already be used on mobile phones with data connections, but using the circuit-switched cellular networks for multimedia data transport allows for better quality of services by reserving bandwidth for the multimedia session. Further, the use of the IP protocol allows for greater flexibility in creating new multimedia services for IMS, similar to the variety of services provided on the Internet.

Presence in IMS is built on top of the SIP (Session Initiation Protocol) event notification framework [6] through the presence event package [3]. Presence is used through devices with software to act as Presence User Agents (PUAs). Notifications are used to keep track of the presence status of all the presentities on a user's contact list. The presence information provides context for possible multimedia communication with a contact in order to help a user to decide when and in what way to initiate a multimedia session. The contents of the presence information are covered in more detail in section 2.4 .

The presence service is illustrated in Figure 2.1. The presentity is the entity (normally a person) sharing their presence status with their friends. The presentity may use multiple devices running software to act as PUAs, allowing the user to publish their presence status. The presence status is sent to the user's Presence Agent (PA) to distribute to watchers which have subscribed to the presentity's presence status. 


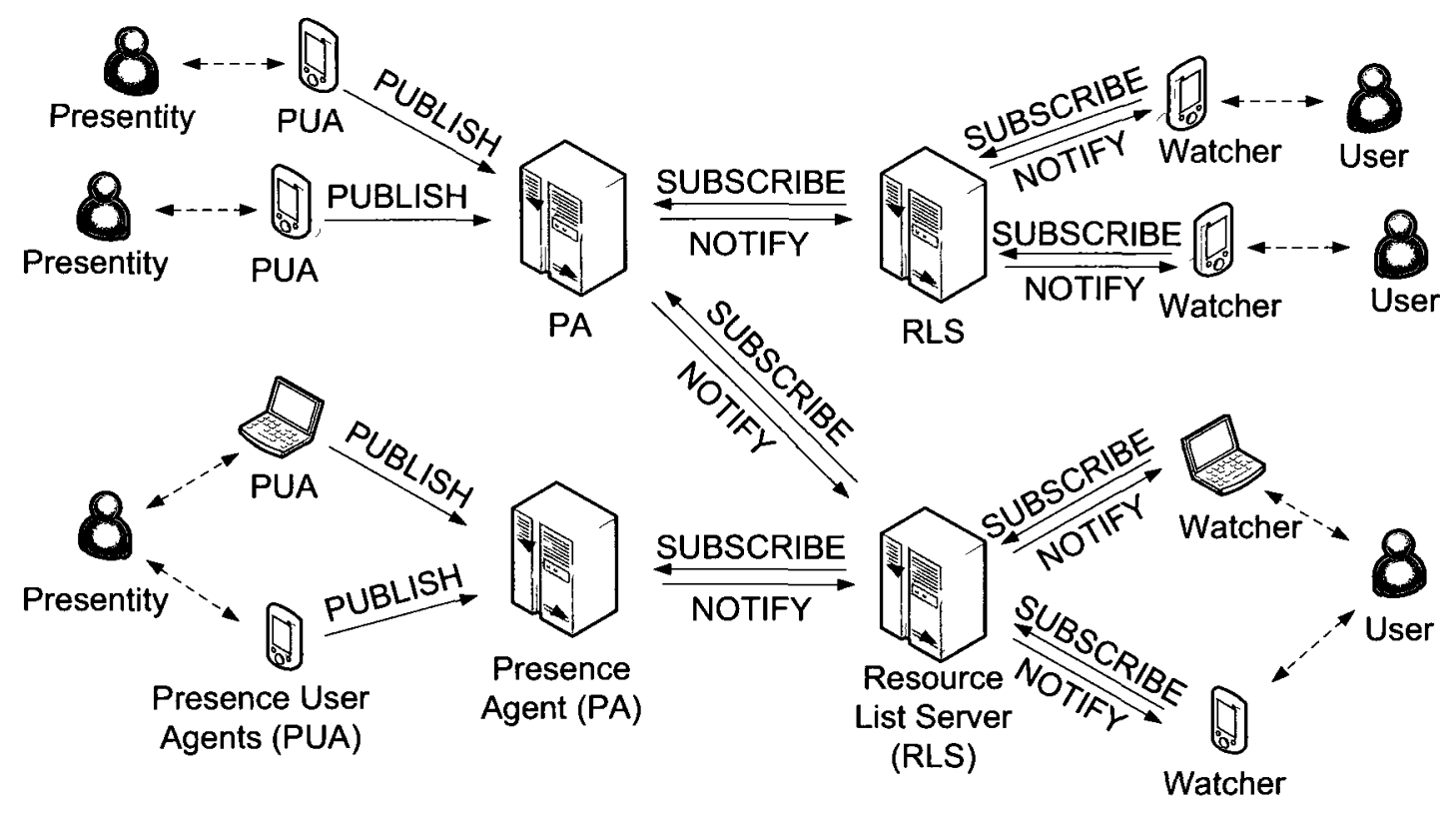

Figure 2.1: Presence Roles

The Presence Agent is needed to provide a single node (network attached device) for watchers to subscribe to, since the user has multiple devices that may not always be connected to the network.

Watchers are any node that subscribes to a presentity's presence status by sending a SUBSCRIBE message to the Presence Agent being used by the presentity. The Presence Agent responds with a NOTIFY message containing the full presence status of the presentity. When the Presence Agent receives a PUBLISH message with the presentity's new presence status, then the Presence Agent will notify all the watchers of the presence status change with a NOTIFY message.

The requested duration of the subscription is specified in the SUBSCRIBE message's Expires header. After a watchers subscription expires, the watcher will stop 
receiving NOTIFY messages from the PA. The subscription duration can be updated with another SUBSCRIBE message specifying a new duration. A duration of 0 may be used to unsubscribe. Such a message will be referred to in this thesis as an unsubscribe message. A SUBSCRIBE message used to delay the expiration of the subscription is referred to in this thesis as a subscription refresh message.

The Presence Agent is always located using the SIP URI that uniquely identifies the presentity (e.g. sip:user@domain.com). The Domain Name System (DNS) is used to lookup the IP address of the Presence Agent by using the domain name (e.g. domain.com) in the SIP URI. The same server may be the Presence Agent for multiple presentities, which are differentiated using the user name portion of the SIP URI (e.g. bob for bob@domain.com). A different PA may need to be contacted for different presentities.

A Resource List Server (RLS), which is defined in [7], can be used to store the user's contact list as a resource list. A user's device can subscribe to the resource list by sending a SUBSCRIBE message to their RLS, which will then send a SUBSCRIBE request to the presence URI (Uniform Resource Identifier) of each presentity on their contact list, as illustrated in Figure 2.2. After a successful subscription, the RLS becomes a watcher for the duration of the subscription, and an initial NOTIFY message is sent with the presentity's presence status. The RLS may then throttle and aggregate the notifications for multiple presentities in order to send a single NOTIFY message back to the user's device which is a watcher of the resource list.

The generic term node is used in this thesis to describe a device because device may play multiple roles. For instance, the RLS is both a watcher of the individual presentities on the resource (contact) list, as well as assuming the role of the Presence 


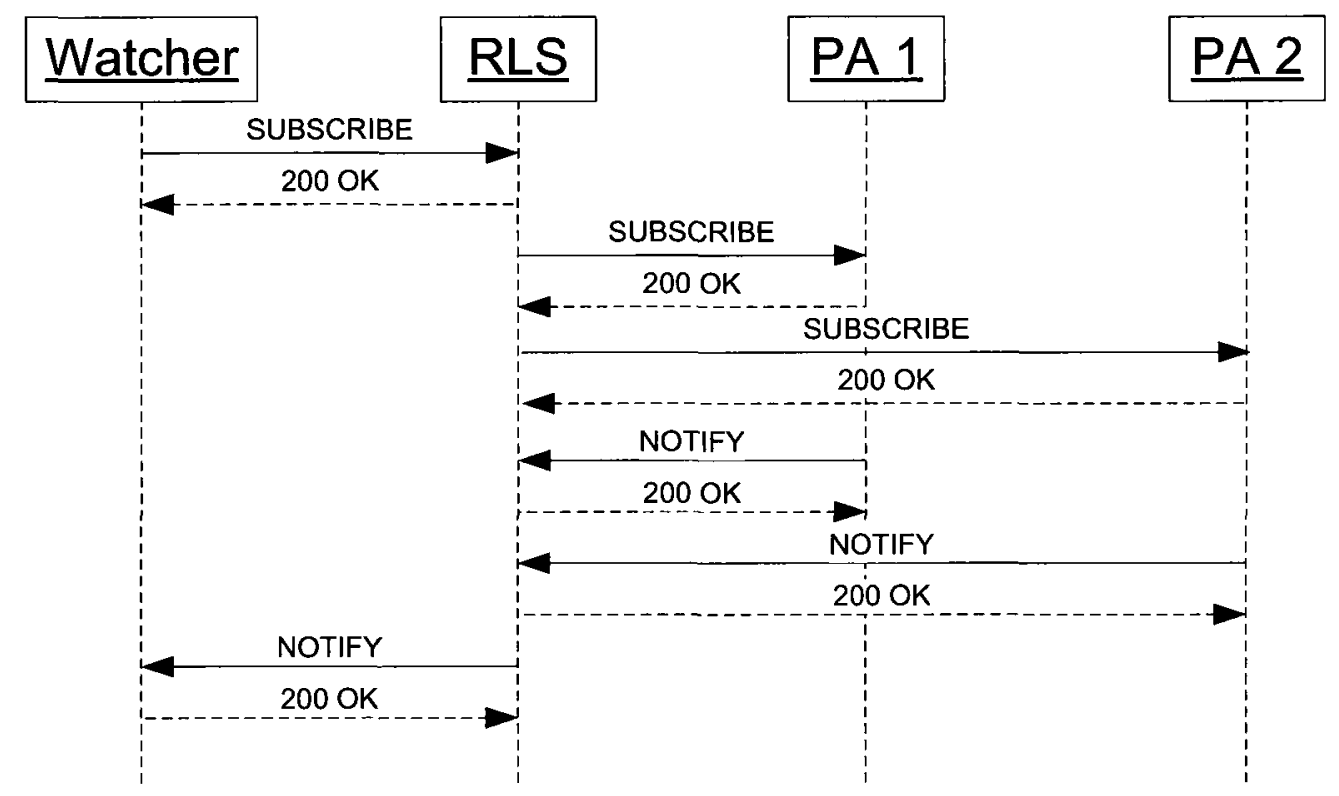

Figure 2.2: Resource List Server Sequence Diagram 
Agent for the resource list for the user's device to subscribe to. A user will generally want to both share their presence status and see presence status of their friends, therefore their device will generally act as a PUA and a watcher of their contact list stored on the RLS. Typically the RLS and PA would be provided by the user's service provider, but these two roles may be co-located at the same server since the RLS already acts as a PA for the user's contact list.

A user's device could directly subscribe to presentities without using an RLS, but then redundant data would be sent through the watcher's access network, which would be less suitable for a mobile device. A watcher not using an RLS can be modeled as an RLS used by a single watcher because of the similar traffic behavior. This thesis will not study the traffic between the RLS and the user's device because the RLS already minimizes the traffic over the user device's access network. Therefore, the watcher can be assumed to be the RLS in the rest of this thesis, since it refers to the watcher subscribed to the status of each individual presentity. Instead, this thesis will study the traffic between the presence servers, the PAs and RLSs, since direct notifications sent by the PA to all the RLS watchers causes congestion in the access network used by the PA.

\subsection{Session Initiation Protocol}

SIP is a multi-layer protocol that does not depend on the transport layer for reliability. This allows User Datagram Protocol (UDP) to be used to send messages, even though UDP does not detect dropped or reordered messages during transport on the network. Instead, SIP defines a transaction layer for detecting dropped messages, and dialogs 
to sequence messages. However, dialogs accept newer messages received before older messages, and reject older messages received after a newer message with an error response. As a result, upper-layer application code must still handle out-of-order packets, dropped packets, as well as crashed nodes.

The transaction layer groups request-response pairs into transactions. Packet loss is handled by retransmitting the request after a retransmission delay. Retransmission stops when the response is received or after the transaction times out. By default the retransmission delay starts at a half a second, doubles after each retransmission up to a maximum of 4 seconds, and the transaction expires after 32 seconds. Responses are saved by the transaction layer in order to resend them when a duplicate request is received. Duplicate responses are ignored, so a 5 second delay after the completion of the transaction is used to let the network be cleared of retransmitted messages.

Transactions between two nodes may be grouped into a dialog for sequencing. The SIP event notification framework uses a dialog for each subscription. Therefore, if notification messages get reordered, the newer presence data will be accepted as soon as it is received, and the older presence data received afterwards will be rejected with an error response. The sequencing of notifications helps prevent accepting old information as new in this case, so old presence data can be safely ignored. When extensions to the notification framework are used, such as the partial notifications which are explained in section 2.4, then an updated NOTIFY message may need to be sent after the out-of-order error response is received.

Crashed nodes can be detected at the transaction layer by another node when it sends a request to it. For the presence service, a crashed watcher will be detected when a NOTIFY message is sent. For the watcher to detect that the node it subscribed 
to has crashed, it will need to send a subscription refresh. Subscriptions are softstate, and expire after the duration specified in the subscriptions Expires header. Subscriptions are refreshed with a SUBSCRIBE message in the same way as the initial subscriptions, using the same event ID so that it is associated with the same subscription.

\subsection{Presence Server Migration}

User mobility is expected for IMS presence. As a result, mobile users may move away from the RLS or PA after they start using these presence servers. This distance creates a delay for all notifications being sent or received by the mobile device. Presence server migration is the process of having the mobile device switch to using a different presence server, typically to reduce the notification delay through selecting a closer presence server.

The event notification framework provides a subscription termination message that allows a PA or RLS to terminate a watcher's subscription before it expires [6]. The subscription termination message is a notify message with a SubscriptıonState header of terminated. This message terminates the subscription and causes the subscriber to re-subscribe using a new dialog with a new DNS lookup to find the server's IP address.

The subscription termination message can be used to migrate presence servers. The PA is addressed by a SIP URI (e.g. "sip:user@domain.com") separate from the SIP URI for the RLS (e.g. "sip:user-buddies@pres.domain.com"), so either presence 
server could be migrated separately by updating the DNS record for the respective domain name (e.g. "domain.com" or "pres.domain.com"). DNS lookups may be cached based on the time to live (TTL) value associated with the DNS record, so a DNS record change may not take affect immediately [8].

Migration of a watcher's RLS would require the RLS to terminate the watcher's subscription with a subscription termination message as described above, and unsubscribe from all the contacts on the watchers contact list. The DNS record for the server must be changed far enough in advance to point so that a cached DNS lookup is not used by the re-subscribing watcher. Therefore, the watcher will re-subscribe to the new RLS, and the new RLS will re-subscribe to the PA for all the contacts on the user's contact list.

Migration of a PA would require the PA to terminate the subscription of all watchers of the presentity after the change to the DNS record for the PA would have taken affect. These watchers of the presentity will re-subscribe, but the updated DNS record will cause them to subscribe to the new PA. The PUBLISH message does not create a dialog [9], so the next PUBLISH message will be sent to the new PA.

There may be significant cost for migrating the PA or RLS based on the size of the watchers contact list and the number of watchers of the presentity, but significant movement of a mobile user may justify the migration. Otherwise, the communication delay between the mobile user and their RLS or PA will result in all notifications sent or received by this mobile user to experience this communication delay. 


\subsection{Presence Data}

The messages for IMS presence are sent using the SIP protocol [10], and the presence data are stored in an XML (eXtensible Markup Language) based format called the Presence Information Data Format (PIDF). PIDF is a minimal specification that only defines a minimal amount of elements, but was designed to be extendible. PIDF only defines in [11] presence data to include an online/offline status, a contact URI (e.g. phone number or SIP address), a human readable note, and a timestamp for when the presence data were last changed.

PIDF alone is not sufficient for commercial applications since it would not even allow a user to specify that they are online yet busy. Four extensions to PIDF that are used for IMS presence are Rich Presence Information Data Format (RPID) [12], Contact Information in Presence Information Data Format (CIPID) [13], the Timed Presence extension [14], and the User Agent Capability Extension [15]. These extensions are backwards compatible, so extra information provided using these extensions is ignored by PUAs not implementing them, and new extensions can be added without breaking existing functionality.

Standard extensions enables devices to properly interpret, display, and update the rich presence data automatically. Presence data may be automatically updated by the device based on user activity, sensor data, or by the presentity manually entering data. For example, a device could update presence status to at work, in a meeting, and in a video conference based on the presentity's Global Positioning System (GPS) coordinates, calendar data and device usage respectively.

Sending the whole presence document wastes bandwidth since only small portions of the presence data will be changed each time they are published. Partial notifications 
save bandwidth by only sending the data that have changed, using a different XML format with additional elements to specify what was removed, added, or modified [5]. SIP provides a mechanism, known as SigComp (Signal Compression), in which the size of the data transmitted can be reduced using compression.

Partial notifications and compression reduce the size of each notification messages without reducing the number of messages. Frequent automatic presence status updates may originate from devices using PIDF extensions, and their notification will be duplicated over an outgoing link for all watchers. Chapter 4 proposes a hierarchical notification protocol that uses network coordinates to build a multicast tree out of watcher nodes, which reduces duplicate notifications on network links without network-layer multicast support.

\subsection{Network Coordinates}

Network coordinates (NCs) are multi-dimensional virtual coordinates calculated for each node in the network to approximate the distance between two nodes in a network without measuring it directly. The distance between two nodes is typically measured using latency of a request-response pair, which is used to update the NCs. The approximate distance between two nodes with known NCs can then be calculated by finding the distance between the virtual positions of the nodes.

In the Vivaldi NC algorithm [16], all nodes start off at the origin. Nodes make measurements between themselves and other nodes to modify their NCs. The algorithm is conceptually a spring-based system; where one could think of there being springs placed between nodes with a rest length equal to the measured round-trip 
time (RTT). Forces are calculated for each measurement between two nodes, and those nodes push away or pull towards each other with a force proportional to the difference between the measured RTT and the one calculated from the NCs. When nodes have the same $\mathrm{NC}$, such as at the start when all nodes are at the origin, a random direction for the force is used, otherwise the direction is parallel to the path between the NCs.

Equation (2.1) is used to calculate the force exerted between two nodes where $L_{\imath \jmath}$ is the measured RTT between node $i$ and node $j ; x_{\imath}$ and $x_{\jmath}$ are the NCs for node $i$ and $j$ respectively. The magnitude of the force is composed of the measured distance subtracted by the NC distance. The direction of the force, $u\left(x_{\imath}-x_{\jmath}\right)$, is parallel to the path between both NCs.

$$
F_{\imath \jmath}=\left(L_{\imath \jmath}-\left\|x_{\imath}-x_{\jmath}\right\|\right) u\left(x_{\imath}-x_{\jmath}\right)
$$

The force will displace node $i$ by $\Delta x_{\imath}$ as shown in (2.2b). The force between the two nodes is weighed based on a weight $w$ from (2.2a), which is calculated using the $\mathrm{NC}$ error $e$ of each node compared to the total NC error for both nodes. The constant $c_{c}$ is used to control how quickly the algorithm converges in order to avoid oscillation of NCs.

$$
\begin{gathered}
w=\frac{e_{\imath}}{e_{\imath}+e_{\jmath}} \\
\Delta x_{\imath}=F_{\imath \jmath} c_{c} w
\end{gathered}
$$

Equation (2.2) requires that each node keep track of their local error $e_{\imath}$, which 
(2.3b) shows to be a rolling average of the error for each sample $e_{s}$, found using (2.3a).

$$
\begin{gathered}
e_{s}=\left|\| x_{\imath}-x_{\jmath}\right|\left|L_{\imath \jmath}\right| / L_{\imath \jmath} \\
e_{\imath}=e_{s} c_{e} w+e_{\imath}\left(1-c_{e} w\right)
\end{gathered}
$$

After enough measurements are made between the nodes, their NCs start to converge to values that can approximate the round-trip time between nodes without direct measurements between them. At the same time the NCs will adapt as the network changes.

Vivaldi NCs can work with normal Euclidean coordinates; however, it was found in $[16]$ that these do not map very well to the structure of the Internet. For instance, most nodes will have a certain network access delay, but the backbone of the Internet can be approximated well using 2-dimensional Euclidean coordinates. Therefore, the Vivaldi algorithm was found in [16] to work best with 2-dimensional coordinates and a height vector to model the network access delay. The distance between two nodes is their Euclidean distance plus their height vectors $x_{h}$ and $y_{h}$, as seen in (2.4).

$$
\|x+y\|+x_{h}+y_{h}
$$

When NCs get pushed from different sides and have nowhere to go in the Euclidean space they are pushed up by increasing their height vector.

NCs have the ability to be reused between distribution trees. Presence would require many different distribution trees, each rooted at a different presentity's RLS since every presentity may be a source of notifications. The process of building a new distribution tree is made simpler by reusing NCs globally within the presence system. 
Pharos [17] is another NC algorithm that uses the Vivaldi algorithm. This algorithm was motivated by the observation that Vivaldi performs well for long links between nodes, but short links suffer from a higher relative network coordinate error. The algorithm improves on the accuracy of the Vivaldi NCs by separating nodes into cluster, then using two separate set of network coordinates, one within the cluster and one between nodes in different clusters.

When a node enters the system, they measure the RTT between themselves and a set of Domain Name Service (DNS) servers with known network coordinates. These measurements are used to initialize their global network coordinates. There is a cluster for each of these sets of DNS servers, and nodes join the cluster for the DNS server that they are closest to based on their global network coordinates.

In the Pharos network coordinates system, nodes continuously measure their distance between any other node in the system. When two nodes measure the distance between each other and they are in the same cluster, then they update their cluster network coordinates, otherwise they use and update their global network coordinates. 


\section{Chapter 3}

\section{State of the Art in Notification}

\section{Dissemination}

The previous chapter covered the standards that have already been adopted for the presence service. The standard IMS presence service uses direct notifications which cause congestion at the network access links of the Presence Agent with duplicate message. These duplicate messages often travel a longer network path than necessary, which could be avoided by using a distributed notification approach.

This chapter discussing existing distributed notification approaches that have already been proposed to solve these direct notification problems, but they have not been adopted or standardized for the presence service, and are not all meant to be compatible with each other. Section 4.1 summarizes the remaining problems that are not adequately addressed by the proposed solutions in this chapter. The following sections of chapter 4 present the alternative solution that this thesis proposes for the notification traffic reduction problem. 


\subsection{IP Multicast}

IP Multicast for IMS has been proposed in [18]. IP Multicast would be an ideal solution to the problem of sending homogeneous notifications to multiple destinations, but IP Multicast has not yet been widely adopted [1]. IMS presence requires communication between presence servers in home networks controlled by multiple service providers, so wide support for IP Multicast would be required in routers between these presence servers. Instead, application-layer multicast only needs a subset of presence servers supporting a new protocol to provide similar benefits.

Multicast-capable routers do exist in certain domains of the Internet, so within those domains it would be beneficial to use this capability. These domains could then be connected using an application-layer multicast as proposed for Island Multicast [19] and Universal IP Multicast [20]. This allows IP Multicast to be used in domains that support it, which increases the efficiency of multicast compared to application-layer multicast. This approach also allows for a transition period where the IP Multicast can be used where available, which provides an incentive for its adoption, before all routers are upgraded with multicast-capabilities. This approach still depends on an adequate application-layer multicast protocol to build the overlay that connects the islands, but may be used with different application-layer multicast protocols.

Island Multicast is designed to use any application-layer multicast protocol to route packets between islands using unicast messages. Each island represents an application-layer multicast node, and each island will have a set of neighbors islands through branches on the application-layer multicast tree. Each island will select a leader. For each neighbor of the island the leader will assign a bridge node to send to the closest node in that neighboring island, which is stored in the node's bridge list. 
The sender of a multicast message will send a multicast message within the island and to all the nodes in its bridge list. A receiver of a multicast message will forward the message as if it were the sender, except will not send the message back from where it was received.

IP Multicast is not currently a complete solution, since application-layer multicast is still needed for maintaining compatibility with non-multicast capable routers on the Internet. The Universal IP Multicast protocol specifies a specific applicationlayer multicast protocol, but this will be discussed in the next section since it could be replaced with a more suitable application layer-multicast protocol.

\subsection{Aggressive Tree Optimization}

The application-layer multicast protocol in Universal IP Multicast [20] has each node find its own place in the tree. A joining node starts out by finding out which node is the root of the tree, and setting that node as the current potential parent. The joining node contacts the current potential parent to get the list of its children. The joining node selects the closest node between the potential parent and its children. If a child was chosen, then it is set as the current potential parent and the process is repeated until the parent node is closer than any children it may have. Afterwards, the current potential parent is sent a join request and the node becomes a part of the tree.

The Universal IP Multicast protocol tries to improve the tree by periodically rerunning the tree joining process from a randomly selected node. If it finds a closer potential parent by re-running this procedure, then it switches to parent nodes. The 
traffic for doing this re-join process to try to improve the tree is the result of an assumption that the traffic from doing so is negligible in comparison with the multicast traffic. However, this would add significant overhead for the presence service which may have low bandwidth traffic.

\subsection{Peer-to-peer}

Peer-to-peer networks have been used for distributing data from a source node to any other node interested in the data, which is similar to the multicast performed in the presence service. The peer-to-peer architecture was even proposed for presence information sharing for FieldCast2 [21]. Network coordinate research has also been done to improve efficiency of file sharing by sending to closer nodes [22].

File sharing differs greatly from the presence service in that a single large set of data is being distributed to a set of interested parties. As a result, it is commonly a requirement to keep the data being distributed even after the source of the data leaves the peer-to-peer network. The large size of the data also allows the data to be split up so that different chunks may be simultaneously downloaded by different nodes at the same time. These reasons make it so a decentralized solution makes more sense that tree structure.

On the other hand, presence data is not large enough to gain any benefits from splitting it up into chunks to distribute separately. The data also is not large enough to justify the overhead of maintaining the peer-to-peer overlay network. The data must be received in real-time, so there is not reason to try to distribute presence information after the presentity disconnects. Therefore, there is no problem with the 
root node being a single point of failure.

The FieldCast2 protocol considers it an advantage to distribute presence over directly between end nodes to avoid the congestion of centralized servers. However, in the IMS architecture the resource list server would still be needed to avoid the need for users to synchronize their contact list between devices, and mobile devices would need to send duplicate notification messages over their limited network access data connection.

\subsection{Minimum Spanning Tree Construction}

A Minimum Spanning Tree (MST) will connect a set of nodes using a set of links. The algorithm starts with no links chosen, then iteratively chooses the shortest link that will connect another node to the tree. The constructed tree allows for an applicationlayer multicast to use the least amount of load on the network.

Network coordinates have previously been proposed as a method of determining the distance of each link for building a MST [17]. This reduces the number of messages needed to determine the distance between each node, but would require nodes to determine their network coordinates and share it with a central node before the tree could be properly constructed.

The problem with using a minimum spanning tree algorithm can be explained using Figure 3.1. The topology of the routers in the example network is a ring topology with duplex links and a single presence server attached to each router. The minimum spanning tree in this case will avoid the longest path between node 1 and node 6 in order to connect all the presence servers with the minimum cost. However, 


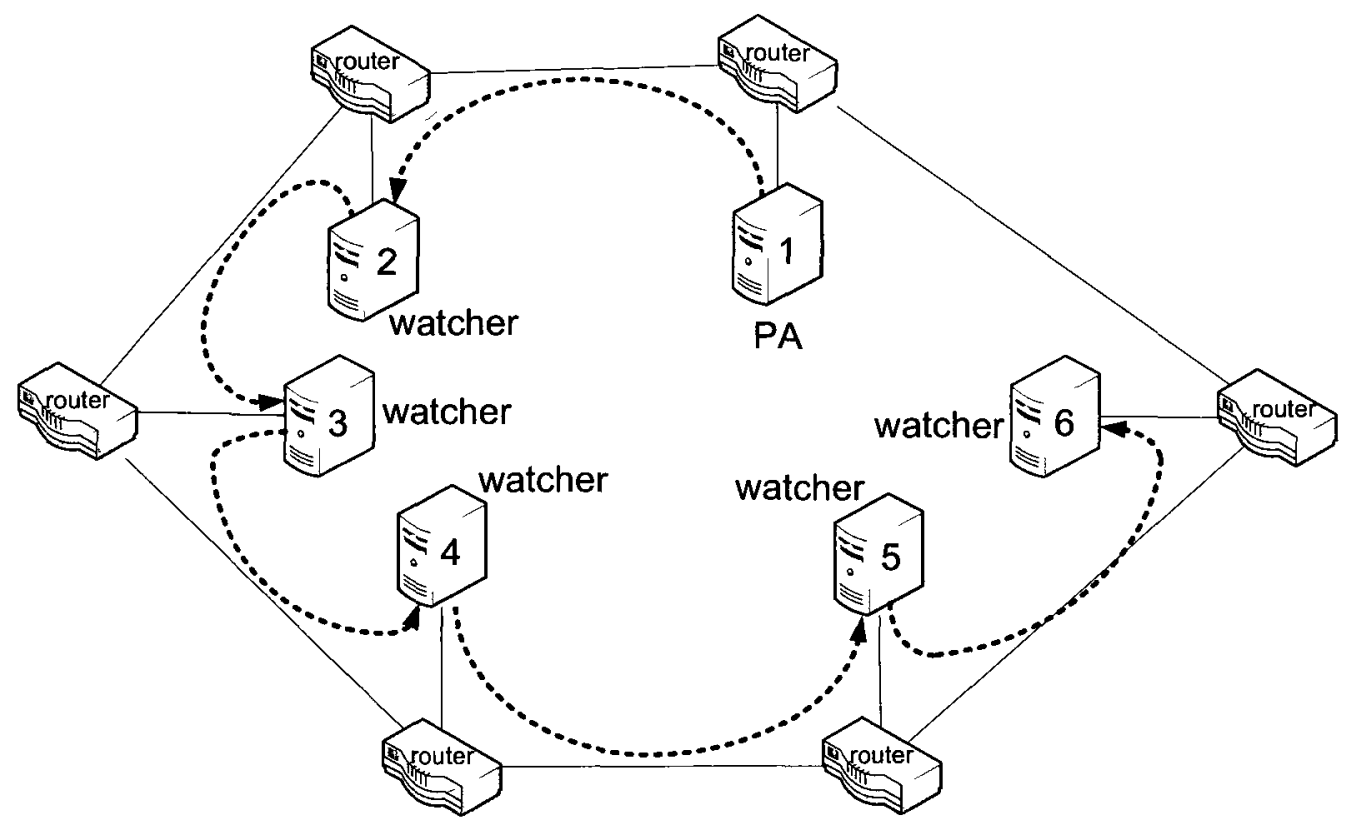

Figure 3.1: Minimum Spanning Tree notification delay problem

if node 1 is the Presence Agent, then the notification will only go around the ring in one direction and cause a much longer than necessary delay for node 6 to receive the notification.

The presence service requires an acceptable notification delay in order to provide a good user experience. The minimum spanning tree does not take into consideration the notification delay that will be experienced by the user, so does not address this challenge.

The presence service also differs from MST construction in that multiple trees need to be constructed from different source nodes, and each may use a different subset of the nodes for each tree. Therefore, a hierarchical tree structure rooted at the source node for each tree would be more appropriate. 


\subsection{Location unaware tree construction}

Tree-based notification dissemination for presence notifications has been previously explored in [23]. Their approach was to set up a distribution tree at a presentity's presence server by dividing the watchers into clusters of at most $K$ nodes where $K$ is the maximum degree in the tree. The clusters are formed by sorting the watchers based on whether the nodes use a radio or wired interface before segmenting them into clusters with at most $K$ nodes each. The clusters are further segmented at each child until a tree is formed.

A problem with this method is that tree notifications still need exactly one notification message to be sent to each watcher to have all watchers notified. The only difference from direct notifications is where these notification messages are being sent from, which could be used to reduce the distance messages travel. However, location information is needed to ensure that the tree is built such that messages are sent between nearby nodes. Instead, notification delay may be greatly increased if the notifications need to travel over long network paths while being distributed down the multicast tree because the tree is built without location information.

Figure 3.2 shows a situation where a short notification from node 1 to node 3 takes much longer than necessary because the distant node 2 is used as an intermediary node. Notifying node 3 first could also have saved bandwidth between the routers on the diagram since only one message would need to pass over them to notify node 2 , instead of the two messages to and from node 2 that are shown on the diagram.

In some cases the intermediary node could be on the other side of the world if chosen randomly. This will result in greater delay and more stress on the network 


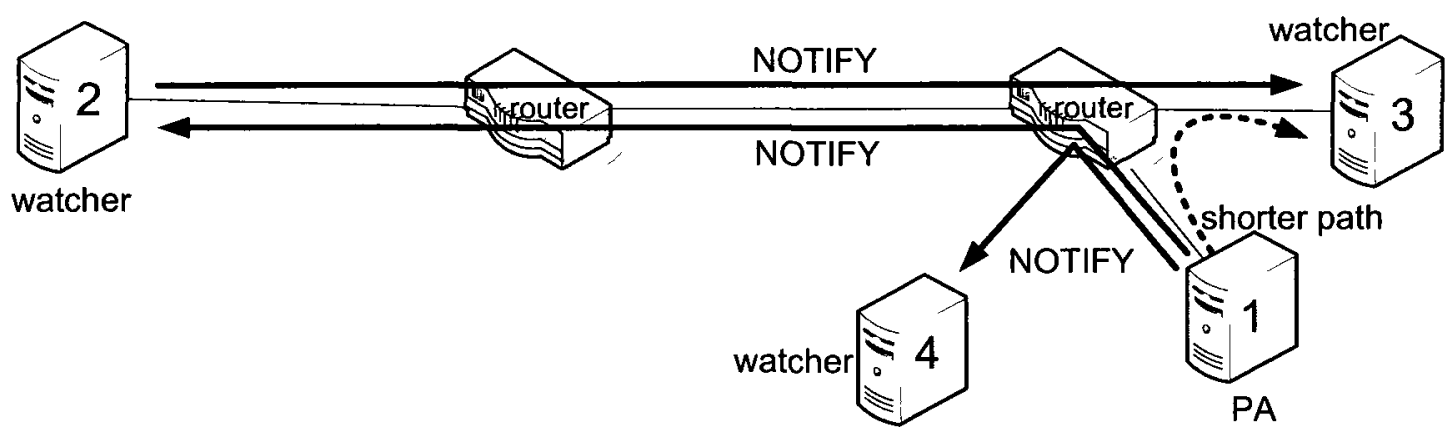

Figure 3.2: Location unaware traffic problem

since notifications will remain in the network for a longer time, either in transit or queued by routers.

\subsection{Temporary Tree Construction}

Group communication within a mobile ad hoc network (MANET) was the target environment for the Location Guided k-ary (LGK) tree [24]. The tree is constructed for each notification, but is not kept due to the frequent changes in the mobile network.

The LGK algorithm constructs the tree similar to the $K$-ary tree used in for presence in [23]. The sender chooses at most $K$ nearest nodes to directly send the message, along with a destination list with the rest of the nodes left to be notified in the subtree cluster. The nodes that are not sent a message directly are added to the destination list of the node to which they are closest. This steps are done by each node that receives the message unless they receive an empty destination list.

Nodes must refresh their multicast group subscription periodically to avoid being removed from the list of other nodes. The is done by sending a refresh to all the nodes using the described tree multicast method. Nodes use these messages to send 
their location information, which was may be a geographical position for the ad hoc network.

This type of solution is suited for a particular type of network which does not relate to the IMS presence service. The presence service provides Resource List Servers (RLSs) to act as watchers on behalf of the mobile user. Therefore the network is more stable.

The destination list being included with all messages is will grow quickly for a large contact list, which will make it less scalable. The refresh messages will also add additional messages since they will need to be sent to all the other nodes in the multicast group. 


\section{Chapter 4}

\section{Proposed Solution:}

\section{Hierarchical Notification Dissemination}

\section{using Network Coordinates}

\subsection{Notification Traffic Reduction Problem}

Notifications generally require sending the same data to a set of subscribers to the updated information. Standard presence protocols use direct notifications, which require sending the same message from the same source to multiple destinations as illustrated in Figure 4.1. This method is inefficient because duplicate data is sent over the same links, and causes congestion on the source nodes network access links. The alternative is to use a network-layer or application-layer multicast solution. This way the shorter available paths may be used to reduce the load on the network.

Network layer multicast requires support by all the routers between the source node and all the destination nodes, but there is insufficient support for using IP 


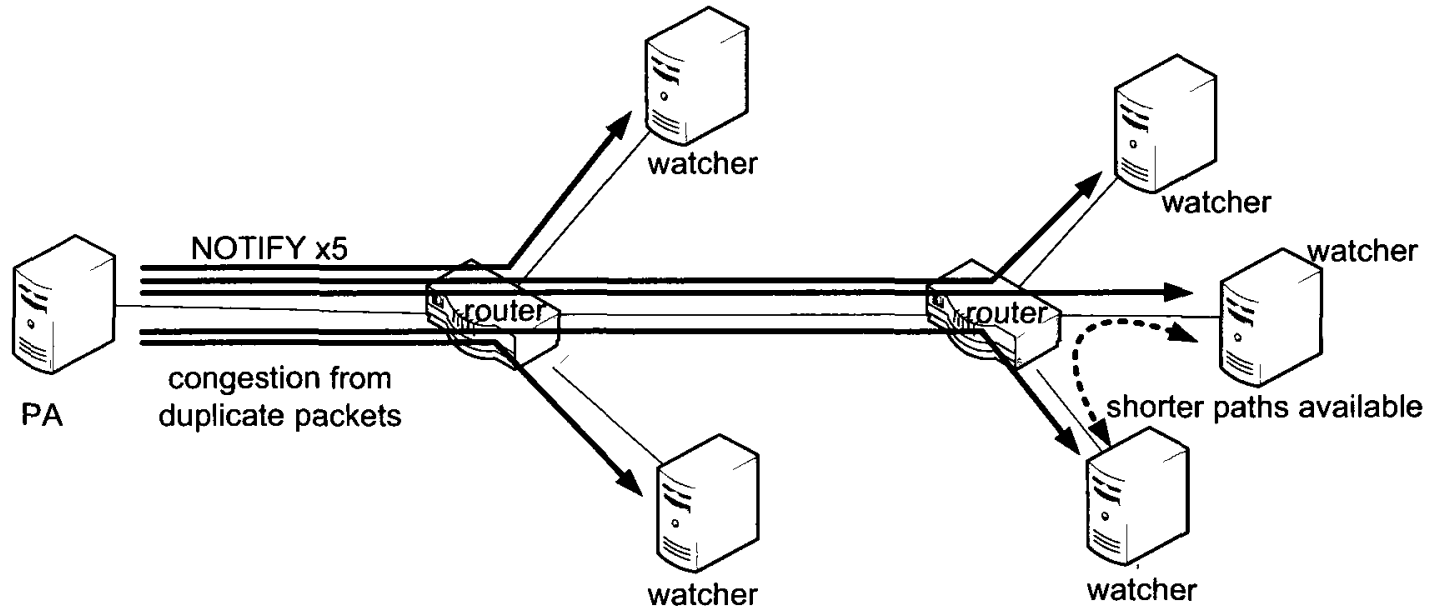

Figure 4.1: Direct Notification Traffic Problem

Multicast over the Internet [1]. Application layer multicast may be used to provide similar benefits, but requires knowledge about the distance between nodes in the network in order to avoid unnecessarily sending messages across the Internet in order to have it received by a destination node close to the source node. A hybrid approach has also been proposed in [19] to use IP Multicast within subsets of the Internet that support it, and use application layer multicast between these subsets where only direct notifications can be used. However, this hybrid approach must first properly solve the problem of inefficient routing at the application layer.

Peer-to-peer approaches to application-layer multicast are unnecessary for notifications, since notifications must originate from the source node, be delivered in real-time. The size of notifications is also not large enough to require splitting the data into pieces for delivery, or to continue delivering after the source node is disconnected. The relatively small size of notifications also requires it to be handled differently from streaming multimedia, since the overhead for building an optimal distribution tree will not be small in comparison to the size of the notification traffic. 
Therefore a low overhead protocol is needed for tree building and maintenance.

The overhead for building a distribution tree must also have a low overhead because if there are multiple sources of notifications, as is the case for presence notifications, and multiple sets of destinations, then multiple distribution trees will be needed for efficient transport. Otherwise, notifications would be sent to nodes who are not subscribed to received them, which would waste bandwidth.

The problem that is addressed by the solution proposed in section 4.2 is that of reducing the effects of the redundant traffic that is needed to distribute notifications without network layer support. The solution to this problem must be applicable to presence traffic, which has multiple notification sources, and different sets of subscribers to each source of notification.

IMS presence traffic provides the motivation for solving this problem due to the need for scalable solution. This scalability is needed to support the large number of mobile communication devices which may integrate which could constantly be using the presence service by integrating it into the contact list. Users of these devices may collect a large number of contacts, and may want to passively provide status updates using the phones usage, sensors, stored user data that the user has chosen to share. These scenarios would increase the number and size of notifications, so a sufficient solution is needed to reduce the effect of this traffic on the network.

\subsection{Proposed Solution}

The redundant data that is sent over the same links for direct notifications can be reduced by using application-layer multicast. Changing the application layer also 
simplifies the deployment, since only the software for the presence servers using the notification framework needs to be changed, rather than needing to upgrade the routers at the core of the network delivering the packets. End user devices do not need to participate in this application-layer multicast since they can continue minimizing their network access traffic by using Presence Agents (PAs) and Resource List Servers (RLSs) as described in chapter 2 .

Application-layer multicast can be performed by organizing nodes into a tree rooted at the source node. The source node sends out a notification request to only its child nodes, and then each non-leaf node in this tree that receives a notification message forwards the message to each of its children.

Tree-based notification still requires the same number of notification messages to be sent; however, the messages are not all sent out from the same node, so the extra load and queuing delay near the source node is avoided. Additionally, the distance that each message needs to travel can be shortened by making sure child nodes are as close to their parent node as possible. Therefore, strain on the network is reduced by minimizing the total number of hops used to notify all the watchers.

Choosing nodes close together requires measurements of the distance between the nodes. To avoid direct measurements between each node, which would have quadratic complexity, virtual coordinates for each node can be calculated and shared. The distance between nodes are then the distance between their virtual network coordinates. Network Coordinates (NCs) are therefore proposed as the method to gather network location information for use in trying to set up an optimal distribution tree.

Vivaldi [16] was chosen as the NC algorithm for tree-based presence notifications 
for its simplicity and wide use as a basis for NC research [22] [17]. Another NC algorithm, such as Pharos NCs [17], could be used in the future if it were found to be better. These network coordinate algorithms were explained in section 2.5.

\subsection{Building the Distribution Tree}

The proposed distribution tree uses a heuristic approach to optimizing the tree in order to reduce the load on the network and the average notification delay. The details on the evaluation criteria are explained in detail in section 5.1.

The distribution tree will start out being empty before a watcher subscribes to the tree. Watchers nodes joining the tree will initially subscribe to the root node to be a child of the root, and receive an initial direct notification with the full presence state. This initial subscription process, as illustrated in the first half of Figure 4.2, is done in the same way even after the tree is constructed. Nodes are not placed elsewhere in the tree until a presence status change occurs. The notification messages for the presence status change are used to assign nodes down the tree, as illustrated in the second half of Figure 4.2.

Notification requests will be used to reassign nodes by specifying a set of new children for the node receiving the message. This allows a new node to be delegated all the way down the tree in a single notification using no extra messages, only a slightly larger message size for the extra information. The reassigned node will receive the new parent notification from its new parent, letting the node know of the new parent assigned to it.

Algorithm 4.1 presents the pseudo-code for the tree building algorithm that is 


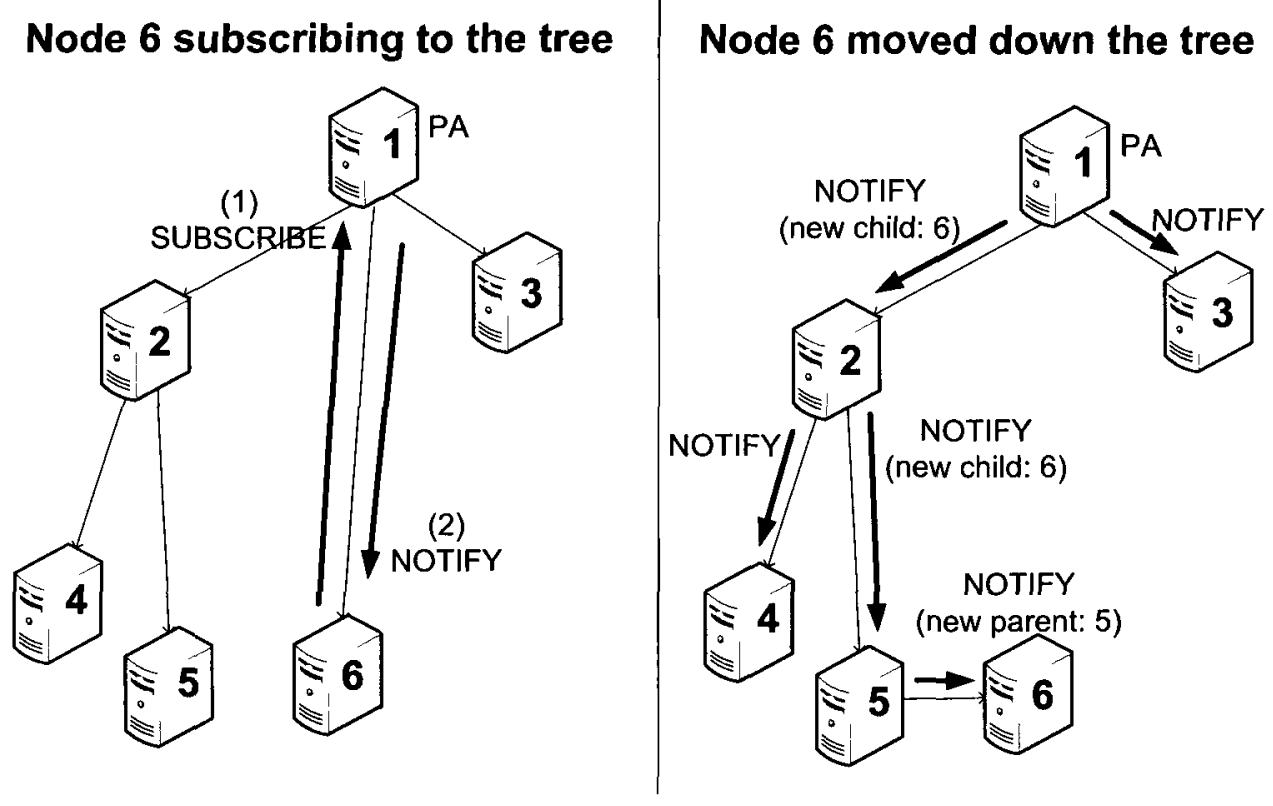

Figure 4.2: Adding a node to the distribution tree

run when any tree node is notified of a presence status change, before notifying its children. The algorithm is used to determine if any reassignments should be made when the notifications are sent. The algorithm starts by separating the children into a list of candidates and a list of nodes with inaccurate Network Coordinates (NCs).

NCs are used to assign nodes to the closest candidate parent node. The NC error is calculated along with the NC position using the Vivaldi algorithm, as explained in section 2.5. The NC error must drop below the error threshold of 0.5 before a node becomes a candidate for being reassigned, or a candidate parent node for another node being reassigned. This error threshold prevents a random tree from developing while nodes have a high NC error.

For each candidate for being reassigned, its parent will calculate the distance between the candidate for reassignment and each of the candidate parent nodes. 
The current parent node and all candidates that have already been considered for reassignment are candidate parent nodes for the current candidate being considered. The node will be assigned to the candidate parent to which it is closest. If the node is closest to its parent, then it will be notified directly, otherwise, it will be reassigned using a notification to the sibling to which it is closest.

The effect of this algorithm is that nodes are effectively grouped into child clusters (clusters of nodes rooted at a child node), and the diameter of the clusters (which is the maximum delay between nodes in the cluster) should get smaller further down the tree.

The order of nodes in the list of children is significant since candidates at the start of the list are considered first, and each candidate only treats candidates preceding it as candidate parents. The reason this is done is because large child clusters are more likely to be at the start of the list and new nodes are likely to be at the end of the list. Adding large child clusters to new nodes is normally prevented using this heuristic, but doing so would increase the notification delay for the all the nodes in the large child cluster by the delay of sending to the new node. It is for this reason that the non-candidate nodes are appended to the end of the list of children after all the candidates are considered for reassignment.

The tree is said to be constructed in a lazy manner because nodes are reassigned on a presence status change rather than during the subscription process. The tree building is also delayed when accurate network coordinates have not yet been obtained. This lazy approach avoids sending extra messages during the subscription process for forming the tree which may be unnecessary if the watcher unsubscribes before a presence status change. It is the presence status change notifications that 


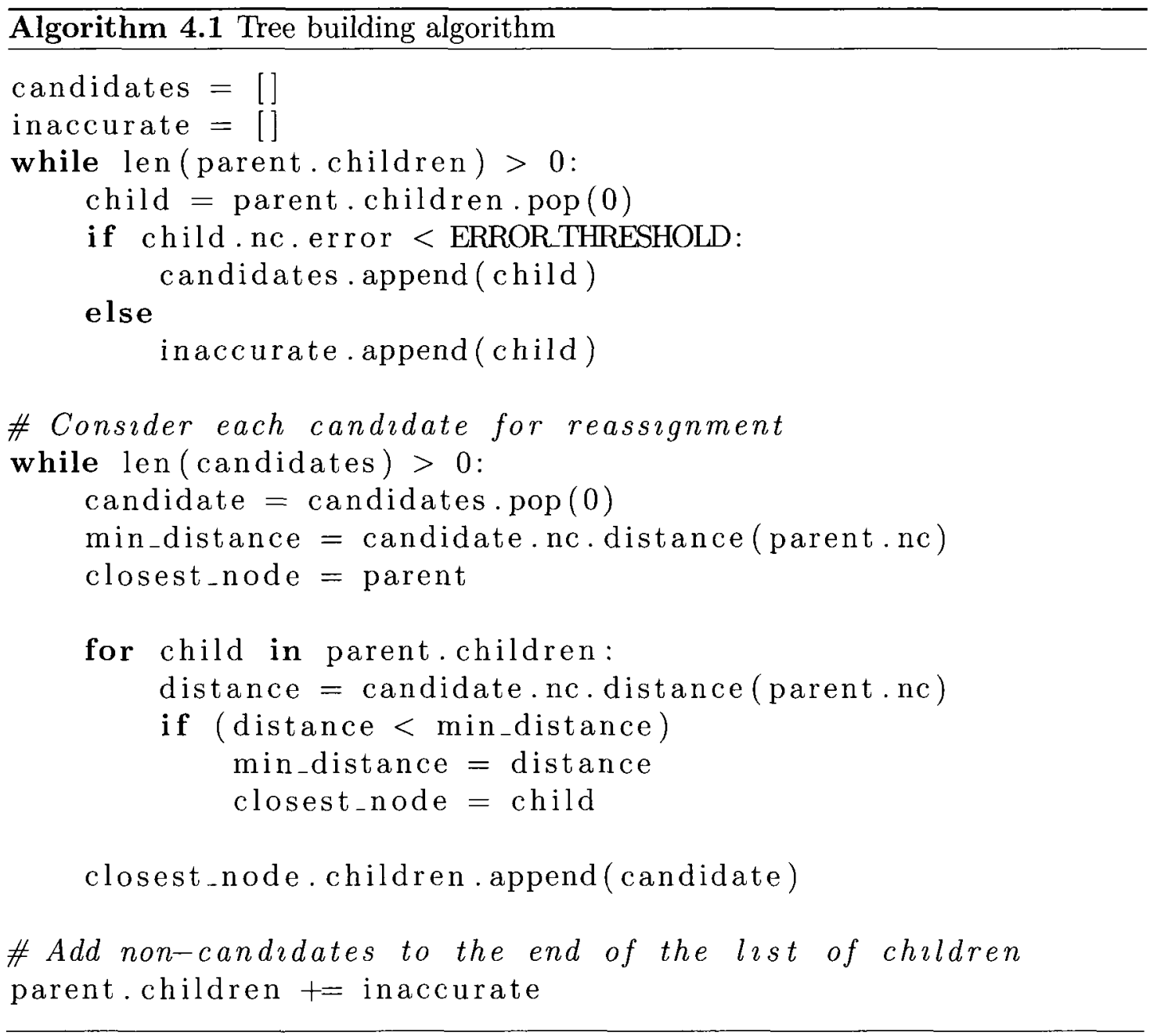


benefit from tree-based distribution, so the tree is formed during this notification process. Lastly, this lazy approach allows notifications to carry the information about tree changes, so no new messages are needed for performing the tree construction.

\subsection{Process of Leaving the Tree}

When a watcher wants to stop receiving notifications, they will send an unsubscribe message to their parent node rather than to the root of the tree. If the node that is leaving has children, then the child with the smallest family delay, calculated using equation 4.1 , will be chosen to take the leaving node's place. This is done by sending an empty notify message to promote a child node at the same time the unsubscribe message is sent to the parent node. The promotion message will specify the node's new parent and may include a list of new children for that node. These steps are shown in the left half of Figure 4.3, with the right half of the figure showing the tree after node has successfully unsubscribed.

Empty notifications are partial notifications that do not specify any presence changes. These will not need to be forwarded to notify children, but will need to be used to let new children know of their new parents using an extra header. The unsubscribe message will need to specify the promoted node as taking the leaving node's place using an extra header. These extra headers are explained in section 4.6.

When a node is being promoted to take its parent's place, a value called the family delay is calculated for all the child nodes. A family refers to a parent node and all of its children. The family delay for node $j$ is calculated in equation (4.1), where $x_{\jmath}, x_{\imath}$, and $x_{p}$ are NCs for the current child being considered, a sibling, and its grandparent 


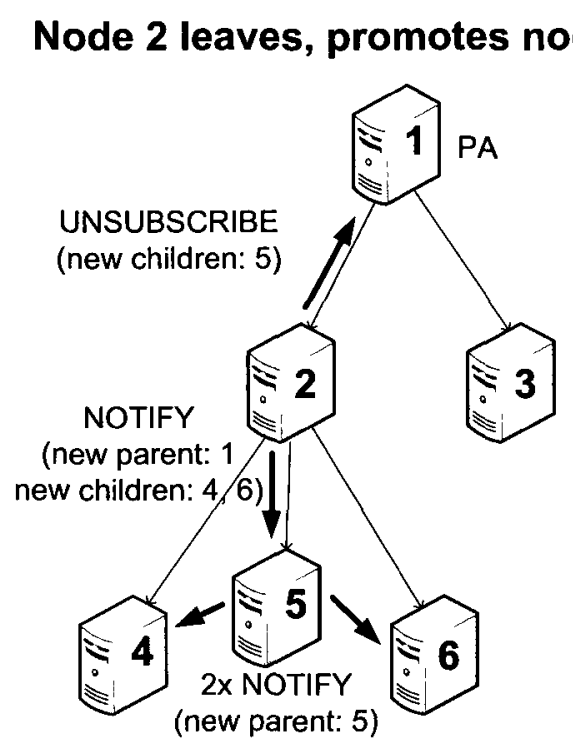

\section{Tree after node 2 leaves}

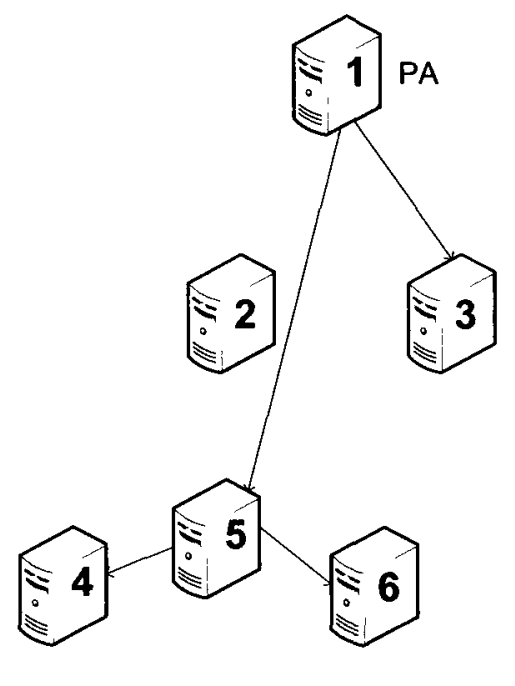

Figure 4.3: Node leaving the distribution tree

respectively. $N$ is the number of candidates for becoming the parent node. The node with the lowest family delay is selected as the new parent of the family.

$$
f a m i l y \_d e l a y(j)=N\left\|x_{j}-x_{p}\right\|+\sum_{i}^{N}\left\|x_{i}-x_{j}\right\|
$$

The weight of $N$ for the delay between the grandparent and the candidate is based on the fact that each child would need to wait for that delay before a message is sent to them. This formula favors reducing average delays in order to choose parent nodes with lower network access delays that are close to the other nodes in the family.

After a node send an unsubscribe request to its parent, the parent may send a notify request before receiving the unsubscribe request. When an unsubscribing node receives a notify request, then node must respond with a pending unsubscribe error with the error code 487 (request terminated). This response lets a parent know that 
it is not accepting any children specified in the request, and lets a new parent know that it is unsubscribing since its unsubscribe message would be sent to the old parent. The unsubscribing node should keep track of the new parent if it was reassigned, since it may still need to promote remaining children.

If a parent receives a request from a child node after having sent a message reassigning it to a new parent, then it must respond with a reassignment error. Error code 480 (temporarily unavailable) is used for a reassigned error to specify the time to wait for a new parent message. However, any new children promoted in an unsubscribe message are still accepted, even when responding with a reassignment error.

An unsubscribing node may need to promote a second node because the first node chosen to promote may be unsubscribing, another node may be unsubscribing and promote one of its children, or a reassignment request sent before unsubscribing might receive a pending unsubscribe response. These situations increase the delay for a node to finish unsubscribing in order to prioritize the concurrent unsubscription of their children. However, the delay in leaving the tree is bounded since they must reject any new children notifications requests. This behavior also prevents nodes from being orphaned when no nodes crash or lose network connection.

\subsection{Maintaining the Distribution Tree}

As mentioned in section 2.2, transactions, dialogs, soft-state subscriptions and subscription refresh requests allow dropped packets, reordered packets, and crashing nodes to be detected. However, in a tree a crashed non-root node could disconnect 
nodes in the tree, a dialogs only provide sequencing between two nodes, and out-oforder responses must be handled in a way that considers the information lost in the new header fields for tree-based notifications.

Sending a subscription refresh request is done in the same way as the initial subscriptions, and uses the same subscription ID so that it is not mistaken as a new subscription. The root node is the only node that receives new subscriptions, but it should not need to store the subscription ID of all the nodes that it has reassigned, since it would not know when those subscriptions end. Therefore, when a node gets promoted to be a child of the root node, the root node may not remember the node. It is possible for the unsubscribe request promoting the node to be dropped, and the concurrently sent notification to the promoted child to be received just before the child needs to refresh the subscription. To avoid confusion, the promoted child must wait at least the maximum transaction time before sending the subscription refresh to make sure their new parent has received the unsubscribe request from the old parent promoting the node.

Any unsubscribe request promoting a node specifies the remaining of time for the subscription. However, just after sending the unsubscribe message the old parent may receive the promoted child's subscription refresh. To avoid the subscription expiring, the promotion request must specify a minimum remaining time for the subscription of three times the maximum transaction time. This will accommodate the maximum amount of time for a node being promoted to receive notification of their new parent, wait for its new parent to receive the unsubscribe request, and then send the refresh request to its new parent.

Nodes may also be reassigned in notification requests sent just before the old 
parent receives the subscription refresh. However, in this case the response to the new parent notification will be treated as a subscription refresh, specifying the amount of time before the subscription expires.

The concurrent unsubscribe and notify requests sent to promote a node may also result in the promoted node sending an unsubscribe request to the new parent before it receives the unsubscribe request promoting the node. The ended subscriptions must be stored by the new parent, even thought it was not previous aware of the node. Otherwise, when the new parent receives the unsubscribe message promoting the node, it could incorrectly believe that it is an active subscription.

When a request is received with a lower sequence number than a previously received request in the dialog, then an out-of-order response is sent. Notification requests receiving this response must resend notification of the new children that have not unsubscribed since the initial request and any part of the presence status change that was not updated by following notifications that were already sent. If no information from the initial request needs to be resent then no notification is needed as a result of the out-of-order response.

Crashed nodes are detected when a transaction times out, which occurs after several retransmissions of the request. A crashed child node can simply have its subscription removed by its parent. A crashed parent will require the orphaned children to start a new subscription to the root node, while keeping its children. If the root node crashes, then there will be no source of new notification and its children will need to periodically send new subscription requests in order to poll the root node until it comes back online. In this case the children may be kept, but a notification indicating that the root node is disconnected must be sent to the children after this 
is detected.

Significant user mobility may trigger the user's PA or RLS to migrate the user to a closer server using the subscription termination message described in section 2.3. If the PA is migrated for tree-based notifications, then the old PA needs to force its children to re-subscribe. Although these direct children of the PA could maintain their children in the same way that is done when the root node crashes, it is the policy for the proposed extension that they propagate the subscription termination down the tree. The relocation of the PA justifies the propagation of the subscription termination down the tree in order to build an efficient distribution tree rooted at the new PA. The next presence status change notification received by the PA may be used to rebuild the notification tree using the tree building algorithm from section 4.3 . RLS migration is done the same way as direct notifications, although the process of leaving the tree has changed according to section 4.4 .

The server migration costs are mostly associated with the forced termination and re-subscriptions, with the presence extension also contributing to an added message size for the following presence status change notification that is used to rebuilt the tree. Therefore, the policy for PA and RLS migration that is used for direct notifications can be used for tree-based notifications without any changes.

\subsection{Compatibility with IMS Presence}

To perform tree-based notification dissemination, a SIP extension is proposed to modify the behavior of the SIP event notification framework in a standard backwards compatible way. This way the extension would be usable for other event notification 
packages in addition to the one for presence.

The SIP extension will be identified by an option tag. Support for the extension is advertised by the watcher when they send a SUBSCRIBE message to a presence server with the extension's option tag included in SIP's Supported header. The response will include the option tag in the Require header if the presence server decides to use this extension. After this point the SIP extension can be used, so semantics of the protocol described in section 4.3 should be followed and extra headers could be used.

The SIP extension will allow messages to include NC information in a NCInfo header, which would store floating point values for the position ( $\mathrm{x}, \mathrm{y}$, height), and the NC error. This will allow NCs to be updated and sent to other nodes to use. The SIP protocol uses request-response pairs so that it can be used over an unreliable network protocol like the User Datagram Protocol (UDP). As a result, nodes sending requests can store the time the request was sent, then calculate the delay when the response is received in order to update the node's NCs. An ACK request could also follow a request-response pair to allow both sides to update their NCs.

In order to build a multicast tree to distribute notifications, extra headers will be needed to move nodes in the tree. The two headers for this purpose would be NewChildren to assign children to a node and NewParent to notify a node that it has been moved to a new parent.

These protocol modifications were implemented for a network simulator as described in section 5.2, along with the standard behaviour for IMS presence that is used as a basis for comparison. 


\section{Chapter 5}

\section{Evaluation of Proposed Solution}

\subsection{Evaluation Criteria}

The protocol extension that was proposed in chapter 4 was evaluated based on reliability, scalability, notification delay experienced by the watchers and the load on the network. The notification delay and network load are measurable quantities. Scalability and reliability are not directly measurable, since they describe the response to an increase in certain inputs or events that are added to the system.

An ideal solution would reduce notification delay and the load on the network. The notification delay must be kept low for a good quality of experience for users, and the load on the network should be kept low to reduce the negative impact the service has on other network traffic. A scalable solution would keep the rate of increase in these quantities low as more users are added to the system in order to increase the capacity of users that can be handled. A reliable solution would properly handle network faults like packet loss and the failure of other nodes in the network while also keeping the notification delay and load on the network low. 


\subsubsection{Traffic Cost}

The load on the network is quantified as the number of packets in transport at any point in time. This quantity will be referred to as the traffic cost. For each packet this includes all the time between the moment the packet is passed to the transport layer for transmission and the moment the packet is received by the transport layer at the destination. Therefore it includes the time that the packet is queued at any router along with time that it spends in transmission in the links between routers.

The traffic cost is used instead of just counting the number of packets sent, because each packet may have a different effect on the network. For instance, packets sent between two computers in the same house will have little impact on the congestion of the Internet as a whole. However, packets sent between continents may require the packet to be sent between many routers that must each process the packet for routing, queue the node for transport over a link, which delays nodes queued after it for transport over the same link or cause those packets to be dropped if the router runs out of memory to queue additional packets. Therefore, traffic cost is a more accurate measure of the load on the network than a packet count.

The traffic cost over a specific interval is the sum of the time each packets spends in transport during that interval. This may be calculated for interval $i$ using (5.1), where $P_{\imath}$ is the set of packets sent during the interval, $t_{p}^{s}$ is the time at which the packet was sent, and $t_{p}^{r}$ is the time at which the packet was received.

$$
\begin{aligned}
& P_{\imath}=\left\{p \mid i \leq t_{p}^{r} \& t_{p}^{s}<i+1\right\} \\
& \operatorname{traffic\_ cost}(i)=\sum_{p \in P_{\imath}} \min \left(i+1, t_{p}^{r}\right)-\max \left(i, t_{p}^{s}\right)
\end{aligned}
$$




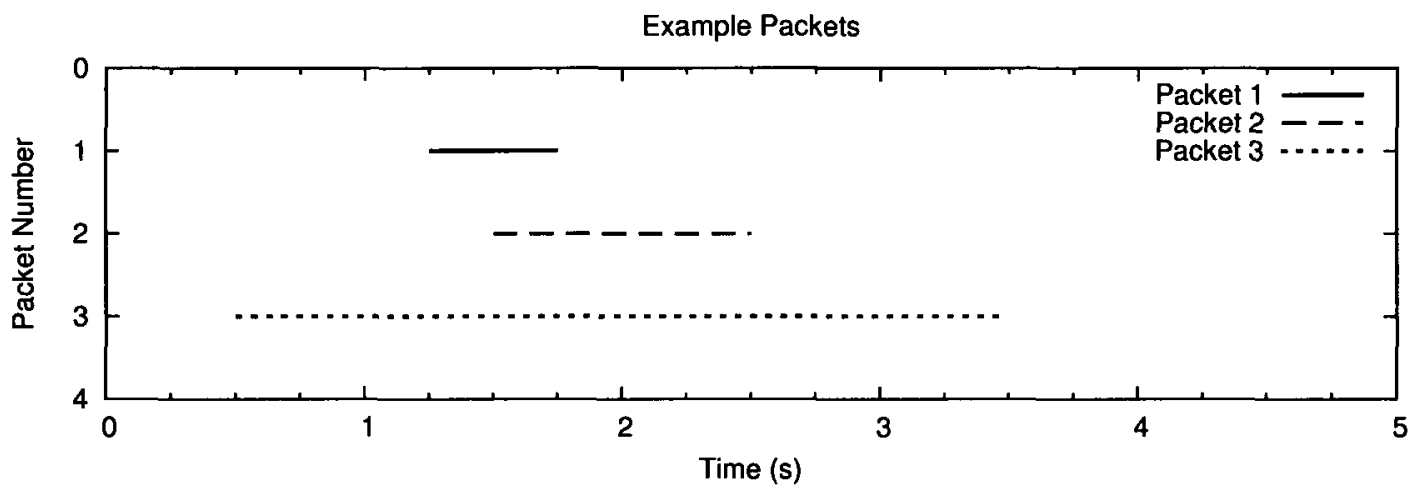

Figure 5.1: Transport timeline for example packets

Some example packets are used to demonstrate how the traffic cost is calculated. Figure 5.1 shows the timeline for three example packets by showing a line for each packet, starting at the time the packet is sent and ending at the time the packet is received. Packet 1 is sent in 0.5 seconds, packet 2 takes 1 second, and packet 3 takes 3 seconds. The traffic cost over the whole time would be the sum of the time each packet takes to be transported, which is 4.5 seconds for these three packets.

A packet's transport time may be divided into multiple intervals. For the example shown in Figure 5.1, if we used an interval size of 1 second, the 2 nd interval would be from time 1 to time 2. The traffic cost for each packet during that interval would be 0.5 for packet $1,0.5$ for packet 2 , and 1 for packet 3 . The traffic cost over that interval is then 2 seconds. This per interval traffic cost is used in section 5.4 to identify the initialization time of the simulation.

The average per interval traffic cost is simply the the average of all the per interval traffic cost values as shown in equation (5.2), where $I$ is the set of intervals being considered. 


$$
\text { avg_traffic_cost }=\sum_{i \in I} \frac{\operatorname{traffic} c_{-} \operatorname{cost}(i)}{|I|}
$$

The sum of the traffic cost over all four intervals in Figure 5.1 was calculated to be 4.5 , so the average per interval traffic cost is 1.125 , which is found by dividing by the number of intervals. The average per interval traffic cost is used in section 5.4 to compare the results based on different inputs to the system. This allows the scalability and reliability of the system to be measured.

The traffic cost is measured for all transport layer packets for the presence service, including dropped packets and retransmitted packets. Packets for other services or protocols, such as a DNS request to resolve the IP of a destination node, are not considered for this measurement.

\subsubsection{Notification Delay}

The notification delay is a measure of the latency experienced by a watcher for the observed presence status changes it receives. The notification delay is only measured when a notification packet is accepted by a node, which causes it to change the state of the presentity that the node stores locally. As a result, the measurement will ignore subscribe, unsubscribe, duplicate notifications, and any responses messages. It will also ignore empty notifications that are used by the protocol extension to reassign nodes in a tree. It will also ignore notifications that are received after a node unsubscribes or loses the state of the subscription due to a node failures, which is described in subsection 5.1.3.

The notification delay for a single notification, $n$, is the delay from the time a 
Presence Agent receives a presentity's publish message, $t_{n}^{r}$, to the time it is received by the watcher, $t_{n}^{p}$. This can be calculated using equation (5.3).

$$
\operatorname{notify} \_d e l a y(n)=t_{n}^{r}-t_{n}^{p}
$$

The average notification delay over a set interval of time can be calculated using equation (5.4), where $N_{\imath}$ is the set of notifications received during interval $i$.

$$
\begin{gathered}
N_{\imath}=\left\{n \mid i \leq t_{n}^{r}<i+1\right\} \\
\text { avg_notify_delay }(i)=\sum_{n \in N_{\imath}} \frac{t_{n}^{r}-t_{n}^{p}}{\left|N_{\imath}\right|}
\end{gathered}
$$

The notification delay for the example packets in Figure 5.1 can be calculated after assuming that all the packets are direct notifications requests sent at the time of the presence status change to watchers that causes them to change their known state of the presentity. In this specific case the total notification delay over the 4 seconds is the same as the traffic cost, 4.5 seconds. The average notification delay would be the total notification delay divided by the number of notifications received, so would be 1.5. The per interval notification delay for the $2 \mathrm{nd}$, 3rd, and 4 th second long interval would be $0.5,1$, and 3 respectively, the delay of each of the notifications received during that interval. The average notification delay during each interval would be the same, because only one notification is received in each interval.

In more complex situations, the delay is not equal to the transport time of the notification request packet. If the packet is dropped, then it needs to be retransmitted, so the delay includes the time it took for the retransmission timer to expire, as well as the time for the notification request packet to be successfully transported to the 


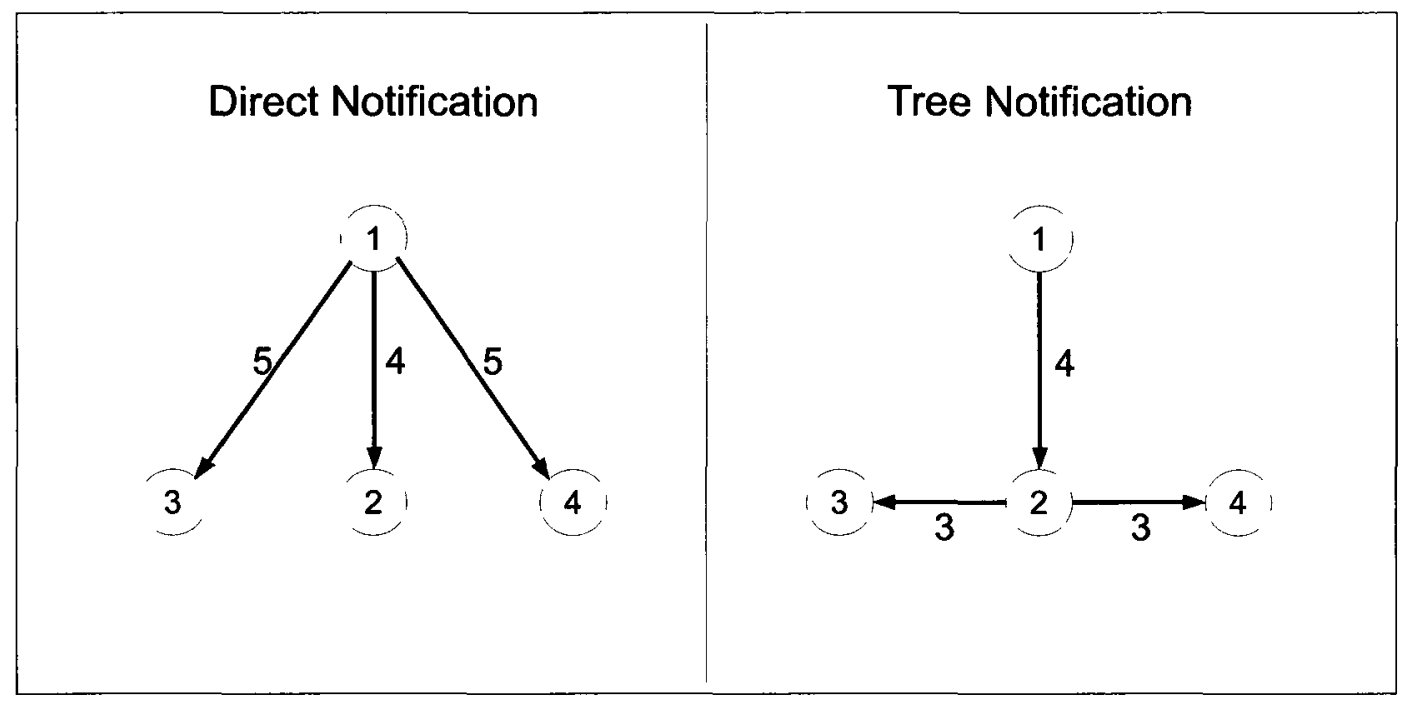

Figure 5.2: Notification Delay Example

watcher.

For tree-based notifications, the notification delay for a child node will be the time for the parent to be notified, plus the time for the parent to notify the child. For example, in Figure 5.2 the direct notifications request packets have the same delay as their traffic cost, which is 4,5 and 5 , for the notifications to node 2,3 and 4 respectively. Tree notifications however require node 2 to receive the notification from node node 1 before a notification can be sent to node 3 and 4 . So the notification delay is 4,7 and 7 for nodes 2,3 and 4 respectively.

The example in Figure 5.2 also shows a trade-off between traffic cost and notification delay. In this situation, the total traffic cost for these notification requests is reduced from 14 seconds to 10 seconds by using tree notifications, but the total notification delay increased from 14 seconds to 18 seconds. 


\subsubsection{Reliability}

Reliability is evaluated based on the traffic cost and notification delay that results from an increase in packet loss and node failures. In addition, the protocol must be able to recover from these event. The process of recovering from these events for the proposed protocol extension was described in section 4.5 .

As described in chapter 2, the presence service uses the SIP protocol, which may use UDP for its transport layer protocol. UDP does not recover from packet loss, the SIP protocol retransmits packets, but this retransmission results in out-of-order packets being received. Therefore, out-of-order packets is not a necessary system input needed to test reliability, so was not used as a system input for evaluating the protocol.

Node failure is necessary to test whether the protocol can handle suddenly unresponsive nodes, which are not able to notify other nodes that they are leaving the system. This could be a result of failure in the hardware, operating system, application, or network access. The recovery time would depend on the type of failure. In the worst case, the node will lose memory of its previous state, so must resubscribe to receive notifications and provide error responses to received requests that refer to an known subscription. Although memory loss may occur if the state is saved to persistent storage, or only network access is lost, the worst case scenario is sufficient to test reliability so this is used for the evaluation criteria. 


\subsubsection{Scalability}

Scalability is also evaluated based on the traffic cost and notification delay resulting from changes in the system input. The scalability of the system was tested by increasing the number of uses. As a result, more presentities exist to send notifications, and more watchers will need to receive these messages. As a result, the traffic cost is expected to increase, but the traffic cost should not increase more due to the use of the protocol extension.

\subsection{Simulation Model and Implementation}

The distributed notification protocol described in chapter 4 was being tested using a simulation. The new protocol extension was implemented for the ns-2 network simulator.

The ns-2 simulation simulates only traffic for the presence service as it is used within the IMS architecture, however it excludes all the other traffic that might occur within an IMS system. It also excludes any supporting traffic such as resolving the IP address of a destination node from a domain name.

The ns-2 simulation uses a star topology, illustrated in 5.3. The center node acts as a router that adds delays to packets in order to emulate the delays on the Internet. The outer nodes in the star topology act as computers connected to the Internet, which send all messages to the router along with a destination for the router forward the message to. The link between the nodes and the router has the minimum delay allowed by ns- 2 of 1 picosecond, allowing the router to add the rest of the delay.

A separate constant delay is used between each node on the network using latency 


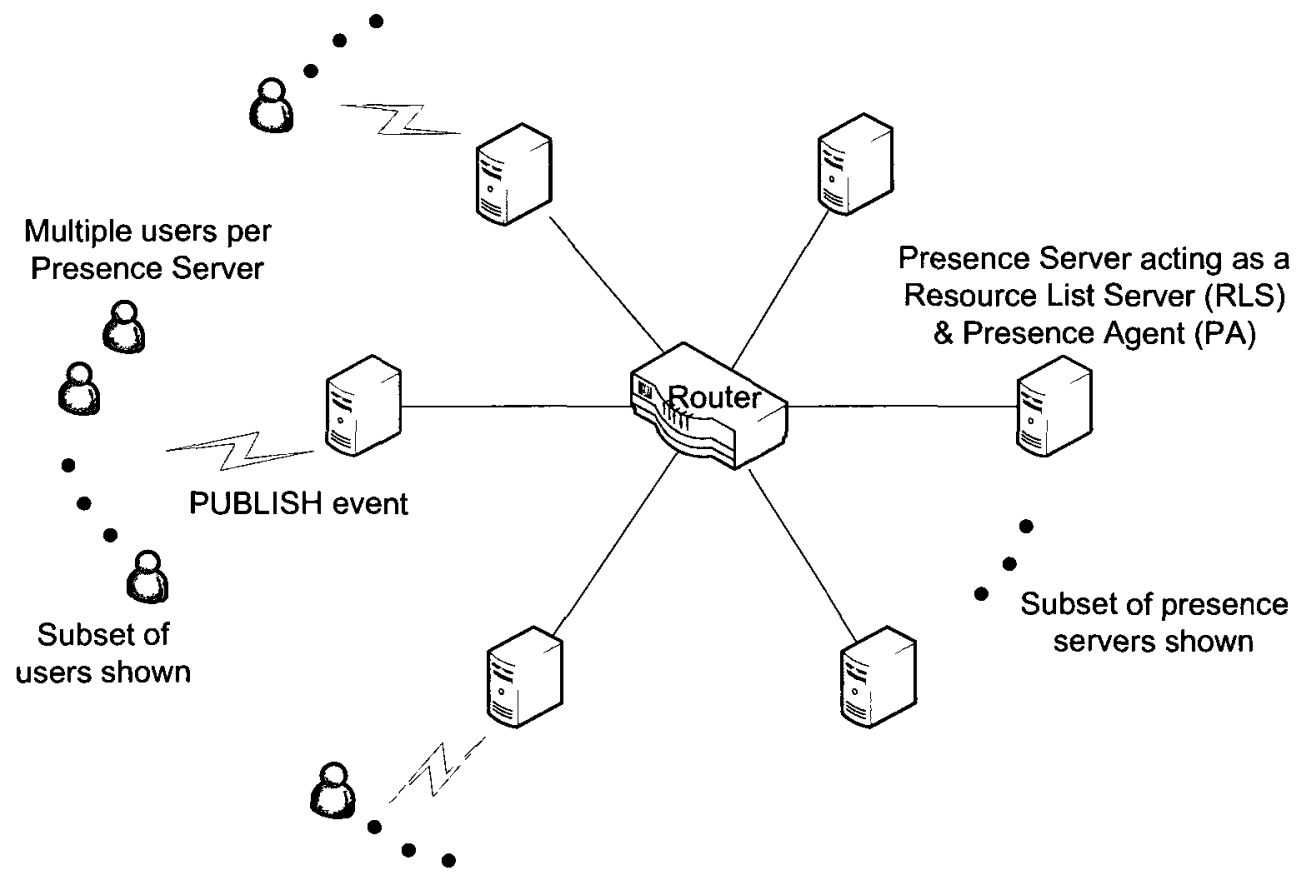

Figure 5.3: Star Topology

data collected from packets sent between actual computers. This data comes from the median round-trip times available from a 72-hour PlanetLab ping trace, described in $[25]$.

The median round trip times were loaded into a two dimensional matrix, such as the one shown in tables 5.1, so that the central router can look up the delay using the source and destination nodes as indices. Sending messages in either direction between any two nodes uses the same delay, so half the matrix is unused by sorting the source and destination indices before using them.

The dataset was missing measurements between some pairs of nodes, so some nodes needed to be removed to have a full half matrix to handle delays between each pair of nodes. A minimal number of nodes were removed by repeatedly removing a 
Table 5.1: Subset of the matrix of millisecond delays between nodes

\begin{tabular}{|c|ccccc|}
\hline Node ID & 0 & 1 & 2 & 3 & 4 \\
\hline 0 & 0.000000 & & & & \\
1 & 52.167936 & 0.000000 & & & \\
2 & 52.187008 & 1.703040 & 0.000000 & & \\
3 & 257.636992 & 286.188032 & 286.194048 & 0.000000 & \\
4 & 6.919040 & 86.464000 & 86.395008 & 253.704960 & 0.000000 \\
\hline
\end{tabular}

node from the set of nodes with the most missing measurements to other nodes in the set until the no more missing measurements remained in the set. This limited the number of outer nodes in the simulation to 197 when using this data set.

All the outer nodes in the star topology act as Resource List Servers (RLSs), sending and receiving notifications on behalf of the users. The users however are not nodes in the network, and the traffic between the nodes and the RLSs are not taken into consideration when measuring the load on the network. However, the proposed extension to the event notification framework does not affect the behaviour of the users or the messages sent between the PUA and their RLS. Instead the users are modeled in the simulation as input events received by the RLS that correspond to the presence status changes sent to the RLS in publish messages.

The simulation takes two different measurements to detect the delay for watchers to be notified and the load on the network. Each of these measurements are saved to a separate log file as a sample per line. Each sample consisting of the time the measurement was made, and value being measured.

The transport time for each packet is sampled by the router in the center of the simulation's star topology. When the router receives a packet and calculates the 
transport time to emulate the delays on the Internet, it also records the current time and the transport delay for the packet to a traffic cost log file. These samples are then used to calculate the traffic cost as explained in subsection 5.1.1.

When a user changes its presence status, the presence status is stored in the simulation with the notification message in order to track the notification delay. When a Resource List Server receives a notification with the new state for a node its watching, then it records the current time and notification delay to a notification delay log file. The notification delay may then be evaluated as described in subsection 5.1.2.

The relationships between users are based on their social relationships, which impact presence traffic patterns. These social relationships are similar to friend relationships on a social network like Orkut, so real world data are used that were collected from public data available from the Orkut social network. The dataset that is being used for the simulation is from the research presented in [26]. Presence status changes are assumed to have a Poisson distribution in the simulation, with the frequency of these events being proportional to the number of users. When a status change event occurs, a presentity is chosen uniformly at random for a status change and their watchers are notified by the RLS. The social network data are used to determine a user's contact list.

Normally the time for sending a packet would depend on the size of data and the bandwidth of the network access link. The time between starting to send two packets could be calculated using equation (5.5). However, IMS has not yet had widespread adoption [27], so actual presence data was not found for properly modeling the data size and type of status change for the simulation. The use case for presence in IMS would differ from the way instant messaging is used on computers, since there 
would be an increased use of the service by mobile devices. Therefore the size of presence data was not used in the simulation, so the simulation uses a data size of 0 and bandwidth value has no effect. This allows a node to send multiple packets simultaneously.

$$
\text { time }=\frac{\text { size }}{\text { bandwidth }}
$$

Packet data sizes would be small for presence due to compression and partial notifications, as explained in chapter 2. Also, sending packets simultaneously would help direct notifications more than tree-based notifications. This is because direct notifications can only send one packet at a time, but distributed notifications enable multiple nodes to each send the same notification packet at the time to different nodes.

When the simulation is being initialized, an RLS is selected uniformly at random for each user, so there may be multiple users mapped to a single RLS. The assignment of users to their RLS is not during the simulation, since as discussed in section 2.3, the message overhead for migrating a user to a new server would only be justified by significant user mobility. The proposed extension should have similar overhead for migrating a user to a different presence server as discussed in section 4.5.

The uniformly random allocations of users approximates real systems, which use load balancing to assign users to lightly loaded servers. The number of users subscribing to each service provider may vary greatly, but service providers will provision the appropriate amount of resources to have the server and bandwidth capacity to support their users. Therefore, the allocation of users will have a greater impact on 
the deployment of hardware by service providers than on the presence service traffic. The presence server is more impacted by the number of presence servers, which will result in larger distribution trees and the traffic to each presence server will be reduced by handling fewer users.

The traffic between the presence user agents (PUAs) and their RLSs are not part of this simulation, but a presentity's status change affects their RLS's behavior. When a presentity's status changes from offline, their RLS makes sure they are subscribed to all the presentities on this user's contact list. When a presentity's status changes to offline, then the RLS unsubscribes from all the presentities that are no longer being watched by their users. Initially all presentities are offline and their network coordinates are reset to the origin with a full error of 1.0.

The presence status changes for each user are assumed to have a Poisson distribution, which assumes that each presence status change occurs completely at random independent any other events. Three presence status states are used in the simulation which represent the online, busy, or offline status of a presentity. This is the minimum number of status needed to cover the three major test cases, which are subscribing when switch from the offline state, notify when switching between online and busy, and unsubscribing when switching to the offline state. The new status change is selected uniformly at random between the two other possible states.

For each user, the mean time between state changes is 20 seconds for the simulation, which is frequent enough to model heavy usage. This will also cause frequent joining and leaving of watchers which will result in additional traffic for tree-based notification dissemination. The reason for this is that direct notification only requires 
an unsubscribe message sent to the source node, but leaving a tree requires unsubscribing from the parent and informing all the children of the leaving node to their parent.

The simulation tests the reliability of the protocols using packet loss and node failure as explained in subsection 5.1.3. Node failure and recovery events are modeled by the simulation to have a Poisson distribution with separate mean rates, which assumes that they occur completely at random, independent of other events. Packet loss is modeled using a binomial distribution, which assumes that the probability of each packet being dropped is the same and independent of all other packets. The probability of a packet being dropped is an input to the simulation that is varied in order to test the reliability of the notification distribution protocols.

In actual networks, higher packet loss would be expected for some nodes with less reliable network access links due to the technology used or environmental factors. However, the correlation with respect to the latency data being used is not available, so would be difficult to model correctly for the simulation. If higher packet loss were experienced by certain nodes, it would result in their network coordinates having a higher error and network access delay (modeled by the height vector with is explained in section 2.5). So this information would be handled by the tree building algorithm and these nodes will more likely become leaf nodes, causing the packet loss to have less of an effect on other nodes in the tree.

Packet loss may also be correlated with certain period of times, resulting temporarily high packet loss. Nodes experiencing this bursty packet loss will temporarily have a high network coordinates error, since this is calculated with a rolling average, and will not be moved or assigned nodes in the tree during this bursty packet loss. 
During this time period the children will experience high notification delays due to retransmission delays, with the worst case being that their subscription refresh does not receive a responded in time, as if their parent crashed causing them to re-subscribe to the root node.

The combined multiple recursive generator MRG32k3a [28] was implemented in ns-2 for its random number generation [29]. This random number generator is used to provide separate streams of random numbers for each independent set of random event needed for a single run of the simulation. Separate substreams provided by the random number generator are used for different replication of the simulation. The period of each substream is $7.6 \times 10^{22}$ random numbers. This allows the result of each different replication to be independent, and the mean and confidence interval can be calculated from multiple replications using the same input parameters for everything but the replication number.

Three independent random number streams are used for in the simulation:

1. For a node choosing random direction vectors during a network coordinate update between two nodes with the same network coordinate. This is mostly used at the start of the simulation in order to have the nodes push away in different random directions.

2. For the router to decide whether or not to drop each packet using the packet drop probability and the binomial distribution.

3. For the event generator to choose input events that are not affected by the behaviour of the nodes in the simulation. This includes mapping users to nodes while setting up the simulation, generating presence status update events, and 
scheduling the next node failure and recovery events. Generating present status updates involves choosing the when the next presence status update occurs, which presentity is updating the presence status, and which state the presence status will be changed to for the presentity.

The first two of the above three listed random number sequences are affected by the behaviour of the protocols. The first one will only be used when using the proposed protocol extension, and the second one will be used at different times depending when the presence protocol chooses to send packets. The third random number sequence is for all the inputs, which are used the same way for simulations with the same input parameters even if they use different presence protocols. This is needed to give a proper comparison between changes to the presence protocol, and easier to replicate a scenario in the simulation by re-using the random number seed and replication number.

\subsection{Simulation Experiment Design}

The simulation that is described in section 5.2 was implemented with certain values parameterized so that they could be specified as inputs to the simulation. These parameters were modified in the simulation in order to test how they affect the evaluation criteria that was described in section 5.1. The user count, presence server count, packet loss rate, node failure rate, and node recovery rate are among these parameters to the simulation.

For each variation of the simulation parameters, the simulation was run multiple times. These set of runs with the same set of parameters are referred to as replications, 
which use a non-overlapping random number sequences. The same random number sequences are used for the same replication number of each variation of the simulation parameters or notification protocol used. Independent random variables are used for each random event which do not overlap with random number sequences for other random variables or other replications.

The results from multiple simulation runs were aggregated taking the Cumulative Distribution Function (CDF), an average, or calculating confidence intervals in order provide more accurate results.

Multiple sets of runs of the simulation were made using different variations of the parameters. This was done in order to determine how changing the standard SIP event notification protocol to using a hierarchical notification protocol would affect load on the network on the network and notification delay experienced by the watchers. The way these measurements were taken is explained in section 5.1.

The way that the simulation is initialized does not reflect the average stable state that it will be in after an appropriate amount of time which will be referred to as the initialization time. During the initialization time for tree-based notification distribution, the nodes will calculate and update their network coordinates and form a tree using their network coordinates. The initialization time only reflects the time when the system first starts or when the system as a whole recovers from a crash. Therefore once the initialization time was found, it was used to filter the results so that they would reflect the steady state of the system.

The traffic cost and notification delay were taken for each time interval individually in order to find the initialization time. For each time interval the results from several replications were averaged. The steady state could then be identified by graphing the 
results, and then an initialization time could be chosen from the start of the steady state.

The following section presents the results of the running the simulation with certain sets of parameter values along with the significance of the results.

\subsection{Evaluation Results}

The simulation results in this section start with finding the initialization time, which was introduced in section 5.3 to describe the time it takes for the simulation to reach a steady state. Further results are filtered using the simulation time. Modifications to the input parameters to the simulation are done for different sets of simulations in order to see the correlation these parameters have on the evaluation criteria. This allows for the scalability and reliability of the proposed protocol extension, from chapter 4 , to be evaluated using the criteria defined in section 5.1.

\subsubsection{Base Scenario}

The first set of simulation runs used 500 users assigned uniformly at random to 60 RLSs, and status changes occur according to a Poisson distribution with a mean time of 20 seconds between status changes for each presentity. The simulation was run for 25 replications, and the results for each interval of one second were averaged. The results from these 25 replications are shown in Figure 5.4 and Figure 5.5.

Figure 5.4 shows that at the start of the simulation, the traffic cost is the same for direct and tree-based notifications. The traffic cost for direct notifications stays around 124, but the traffic cost for tree-based notifications drops over the first 100 


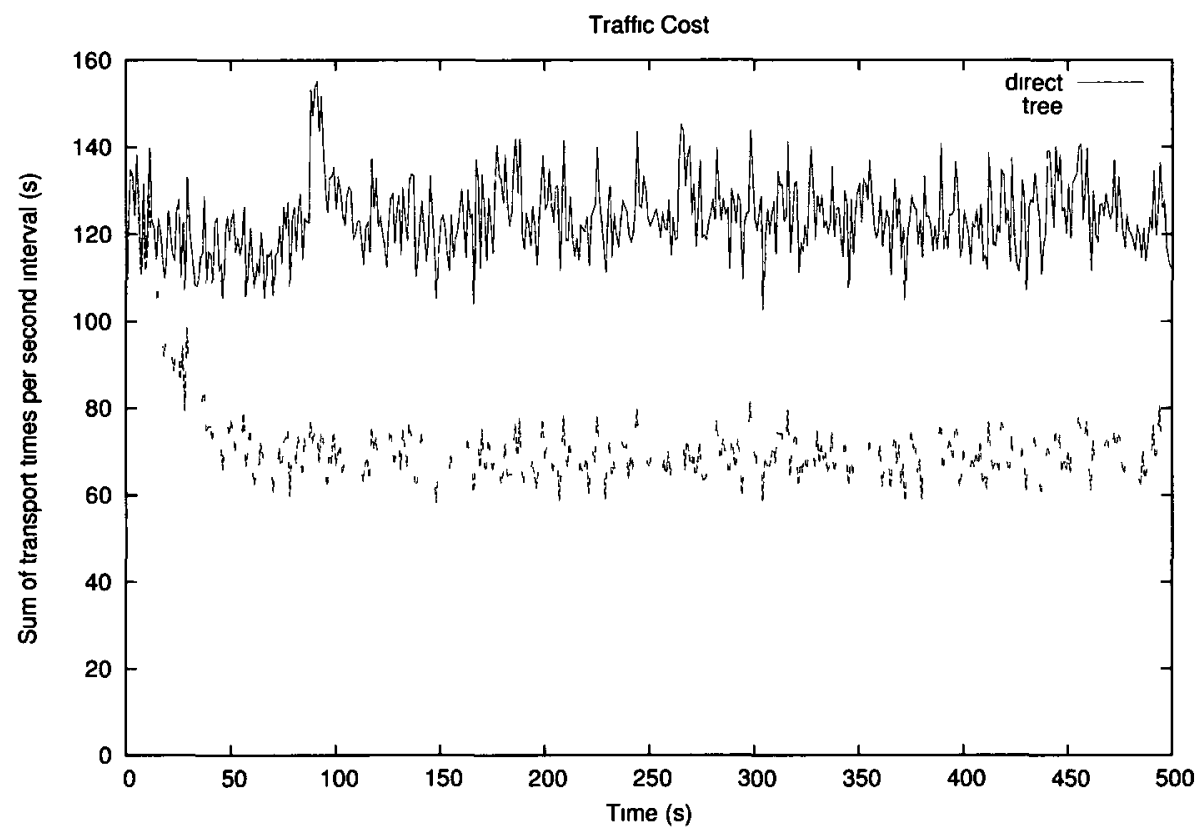

Figure 5.4: average traffic cost per time interval

seconds before stabilızing around near 69 for the rest of the simulation The change in traffic cost over the first 100 seconds illustrates the initialization time which does not reflect the steady state of the system

The effect of the initialization time can also be seen in Figure 55 for the notificatıon delay The delay for direct notıficatıons stays near 010 seconds throughout the simulation For tree-based notıfications the delay starts out at the same value, rises during the first 100 seconds of the simulation, then the notıficatıon delay stabilizes near 013 seconds for the rest of the simulation

For tree-based notifications the simulation is initialized with all nodes having no network coordinate information, so their network coordinate error is initialized to 10 to represent $100 \%$ uncertainty During the initialization time the network 


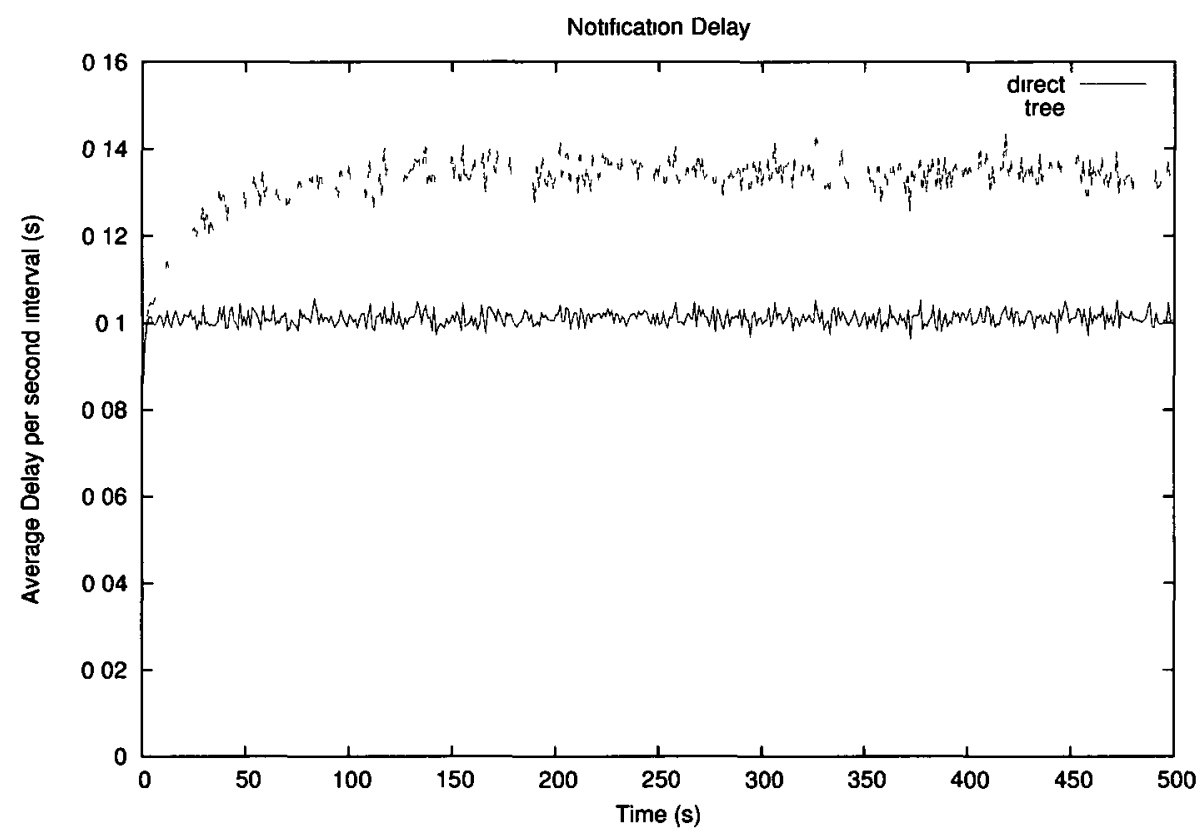

Figure 5.5: average notıficatıon delay per time interval

coordinate are not accurate enough to produce a tree with short branches to keep nodes close to each other, so direct notıfications are initially used by introducing a network coordinate error threshold. When the calculated network coordinate error for a node drops below the chosen error threshold of 05 , then the node may then be assigned children or be assigned as a chld of another node This explains why the traffic cost and notıfication delay is consistent with direct notıficatıons and the start of the simulation

The initialization time was chosen to be 100 seconds based on the results of Figure 54 and Figure 55 The following results ignore the initialization time in order to calculate an average over the rest of the simulation for the chosen measurements, 
Table 5.2: Average steady-state values for base scenario

\begin{tabular}{|c|c|c|c|}
\hline metric & direct & tree & $\%$ change \\
\hline notification delay & $101.1 \pm 0.9 \mathrm{~ms}$ & $134.4 \pm 1.8 \mathrm{~ms}$ & $33.0 \pm 2.7 \%$ \\
traffic cost & $124.4 \pm 2.1 \mathrm{~s}$ & $68.7 \pm 1.1 \mathrm{~s}$ & $44.8 \pm 2.6 \%$ \\
packet count & $1222 \pm 15$ & $1307 \pm 18$ & $6.9 \pm 2.7 \%$ \\
\hline
\end{tabular}

which represent the steady-state of the system. The results for the same set of simulations can be seen in table 5.2 .

Table 5.2 shows that, in the absence of network errors, tree-based distributed notifications can cut the network traffic by $44.8 \%$ compared to direct notifications when the simulation reaches a steady state. The packet cost was also measured using a cost of 1 for each packet, ignoring the duration each packet spends in the network. Distributed notifications only required a $6.9 \%$ increase in packets being sent, which shows that the protocol extension requires very few extra packets to be sent, and that these packets are sent over shorter paths in the network.

Additionally, the results show an increase in the average notification delay by $33.0 \%$ switching to tree-based notification from direct notifications. The average notification delay remains at only 0.134448 seconds, but the notification delay experienced by each node will vary based on their network access delay. It is not possible for a node to experience a notification delay smaller than their network access delay, so each node will experience a different notification delay.

Figure 5.6 shows the Cumulative Distribution Function (CDF) of the notification delay from the same samples used to generate Figure 5.5. The graphed CDF shows that half the distributed notifications will have a delay of less than 0.136 seconds, and $99.8 \%$ of will have less than a half a second delay. This delay would be difficult 


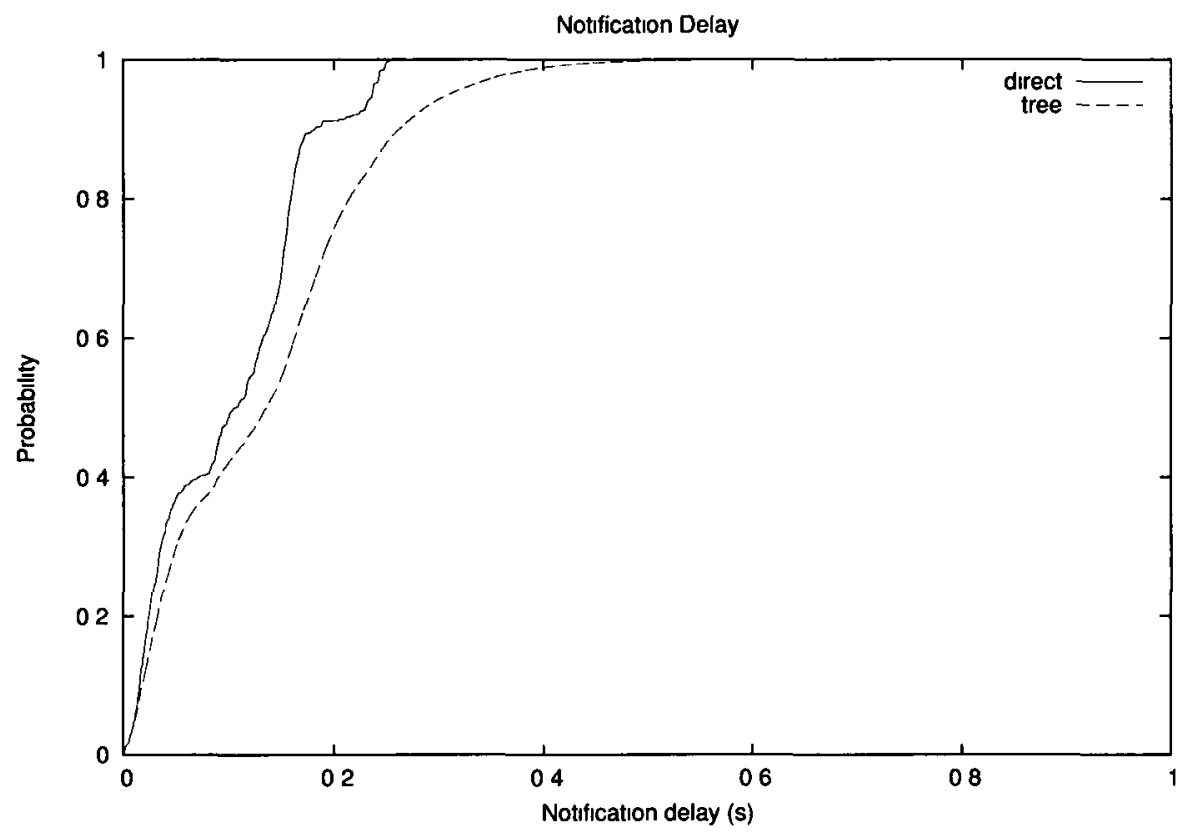

Figure 5.6: $\mathrm{CDF}$ for notification delay

to notice by a user of the presence service because it is not directly a part of an interactive communication.

\subsubsection{Reliability}

The reliability of the distributed notification protocol was tested using packet loss and node failure. Separate sets of simulation runs were used to test packet loss independently from node failure in order to see the effect each parameter has on the measurements.

The rate of packet loss was varied for the results shown in Figure 5.7 and 5.8. The rest of the parameters are the same as for the previous results which were used to find the initialization time, but with the initialization time filtered from the results. 


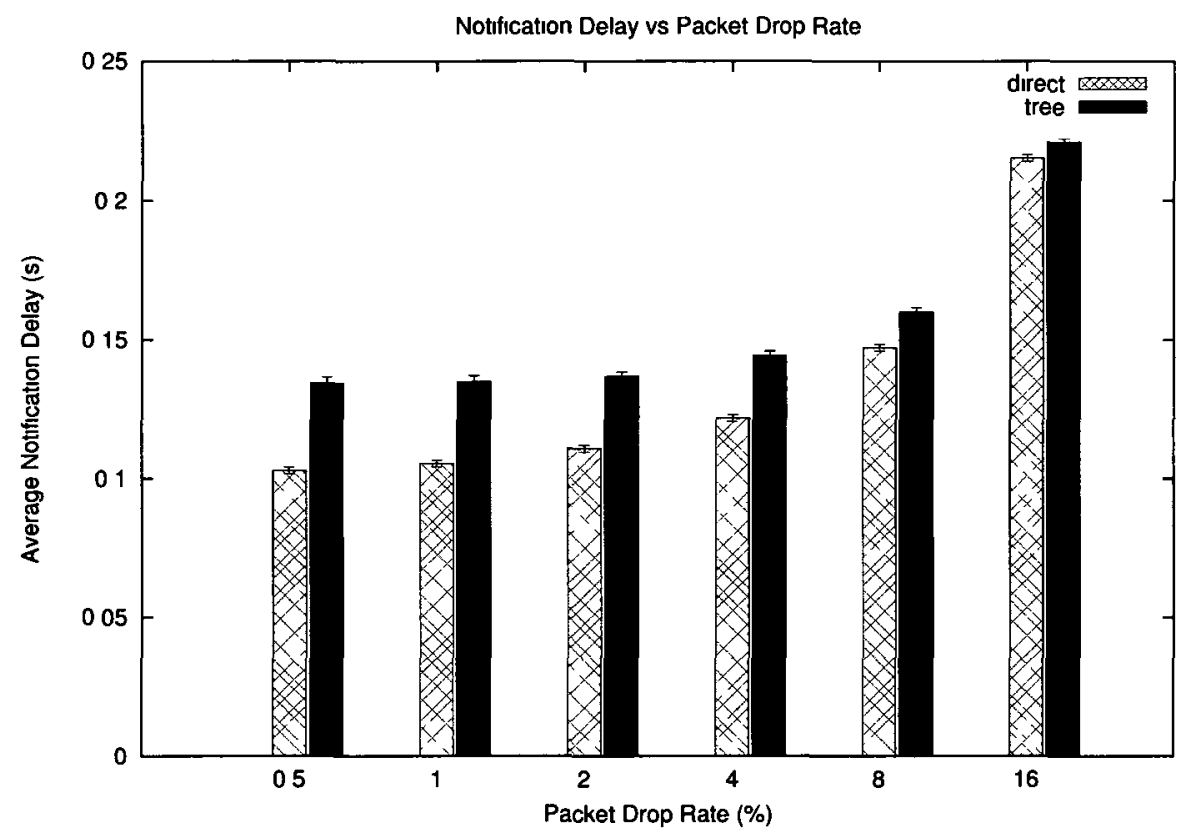

Figure 5.7: average notification delay

These figures show the $95 \%$ confidence interval using error bars for the results from the set of replications at each variation of the drop rate. The packet drop rate is doubled for each variation in order to test a wide range of packet drop rates.

The average notification delay in Figure 5.7 shows that there still remains to be an additional delay for using tree-based distribution of notifications. The retransmission delay from the dropped packet were expected to add delay for both protocols, which can be seen on the graph where the notification delay increases as a greater percentage of packets are dropped. However, even with a large rate of $16 \%$ of the packets being dropped, the average notification delay still remains less than a quarter of a second for both direct and tree-based notification distribution.

The traffic cost is shown for each variation of the packet drop rate in Figure 5.8. 
These results indicate that the packet drop rate has a greater negative impact on treebased notification distribution than for direct notifications, but a large rate of nearly $16 \%$ of packets being dropped are needed to cause tree-based notifications produce more traffic.

When packets are dropped, SIP will resend the packet automatically using the transaction layer until a response is received for the transaction. By following the SIP protocol, the extension to the SIP event notification framework is not able to record the time that the request is retransmitted, and is not able to detect whether the response it receives is a response to the original or retransmitted request. The effect of this is that the network coordinates will be inaccurate, which will prevent trees from being formed, or cause trees to be formed using incorrect network coordinates. This would explain how the direct and tree notification protocols have their results converge near the $16 \%$ packet drop rate.

The packet drop rate was restored to zero percent and the node failure rate was varied in order to produce Figure 5.9 and 5.10. The rest of the results were kept constant from the previous mentioned simulations. The mean time to failure for each node started at 10000 seconds (2 hours 47 minutes), and then reduced by half for each variation. A node recovery rate was used with an average of 50 seconds for a recovery with a Poisson distribution. Node failures are assumed to cause the node to forget all data about the nodes network coordinate, subscription state, and child information for the tree. Node failures could be caused by an application crash, system crash, or hardware failure.

Figure 5.9 shows the affect that node failures have on the average notification delay. The graph shows that infrequent node failure causes large increases in the 


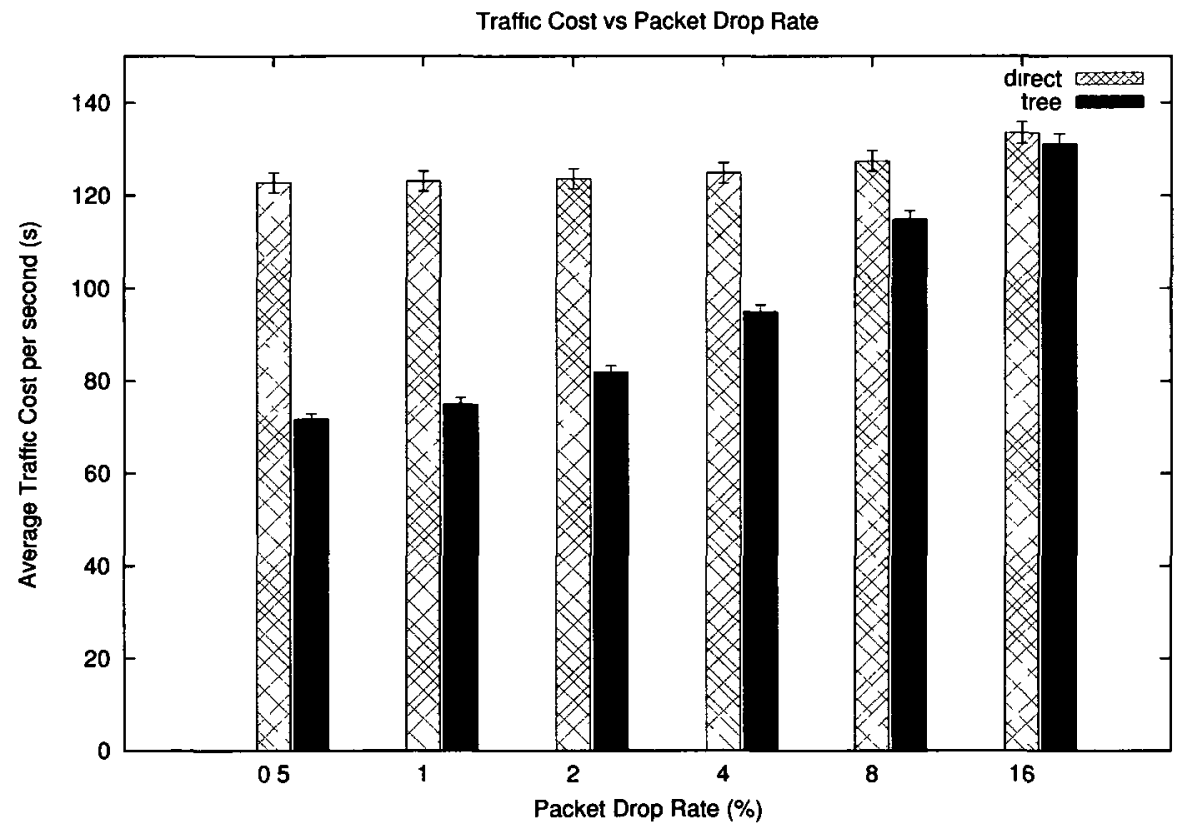

Figure 5.8: average traffic cost

average notification delay for tree-based notifications, but after node failures become frequent enough they start to reduce the notification delay. Direct notification do not show the affects of node failure, since after a node crashes it will stop sending notifications, so there will not be samples of notifications to show that the node is not receiving notifications.

The average notification delay for tree based-notifications appears to be greatly affected by at least two factors in order to have the notification delay increase for infrequent notifications and decrease for frequent notifications. The reason for the increase is that children are briefly orphaned when their parent node crashes, which will not be detected until a transaction timeout. This will cause delays that are not present for direct notifications, and will increase the delay as crashes become more frequent. However, crashed nodes lose their network coordinate information, so after 


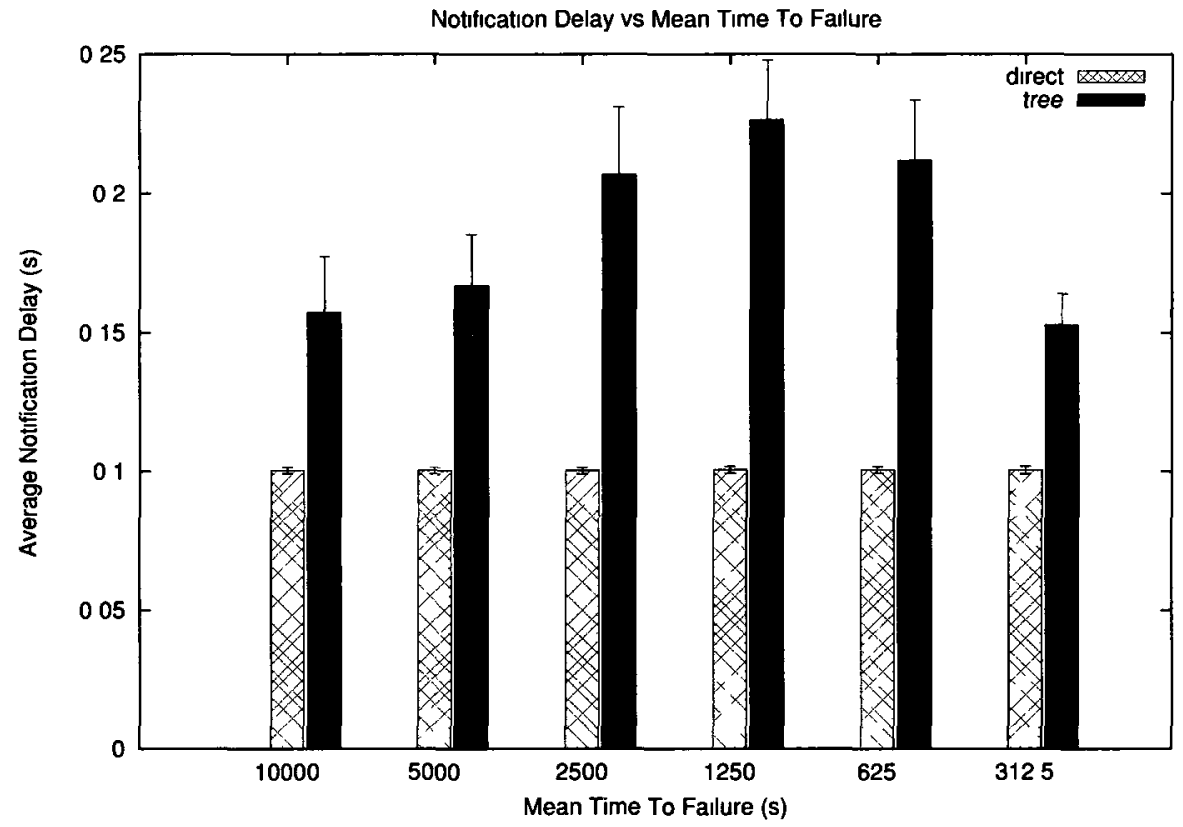

Figure 5.9: average notification delay

recovery they will not be assigned children, or assigned as children in the distribution tree until their network coordinate accuracy increases. Frequent crashes would thus slow down the formation of trees, which would explain why the notification delay starts to converge towards the average notification delay for direct notifications as crashes become frequent.

The results from varying node failure rates were also graphed for the traffic cost using the samples from the same simulations as for notification delay. The results are shown in Figure 5.10. The graph shows that node failure rate causes only a small increase in traffic cost for direct notifications, but frequent node failures have a significant impact for tree-based notifications. However, the traffic cost without node failures is reduced in half for tree-based notifications, and takes a very frequent failure 


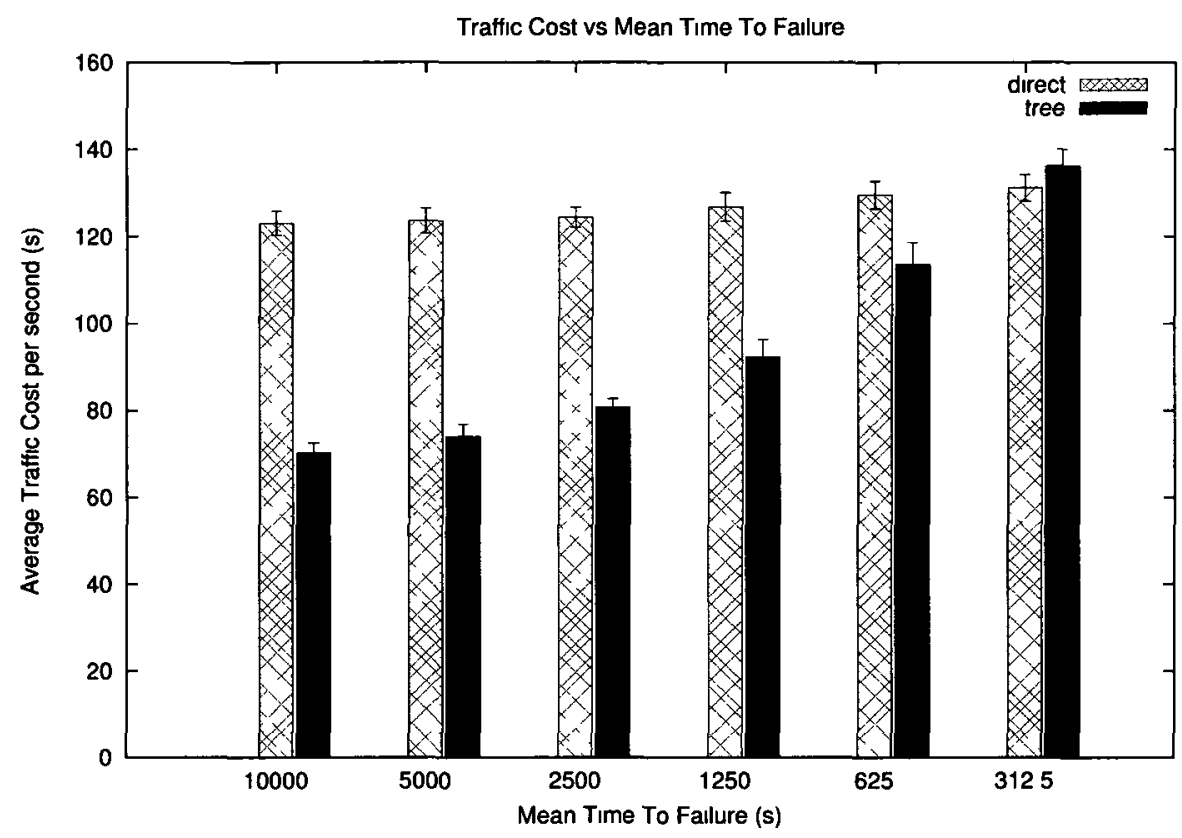

Figure 5.10: average traffic cost

rate for the traffic cost to be comparable between the two notification protocols.

The traffic cost converges towards a similar value for frequent node failures just as the average notification delay does for the same simulation. Therefore the lack of accurate network coordinates may also be increasing the traffic cost.

The introduction of faults into the simulations was necessary to ensure that the protocol extension would work properly under these conditions. Although the high rate of node failure and packet loss were tested, they would not be expected in a production server. These failures do negatively impact the notification delay and traffic cost, but demonstrate that tree-based notification dissemination is able to handle the faults with reasonable penalties to these measurements. 


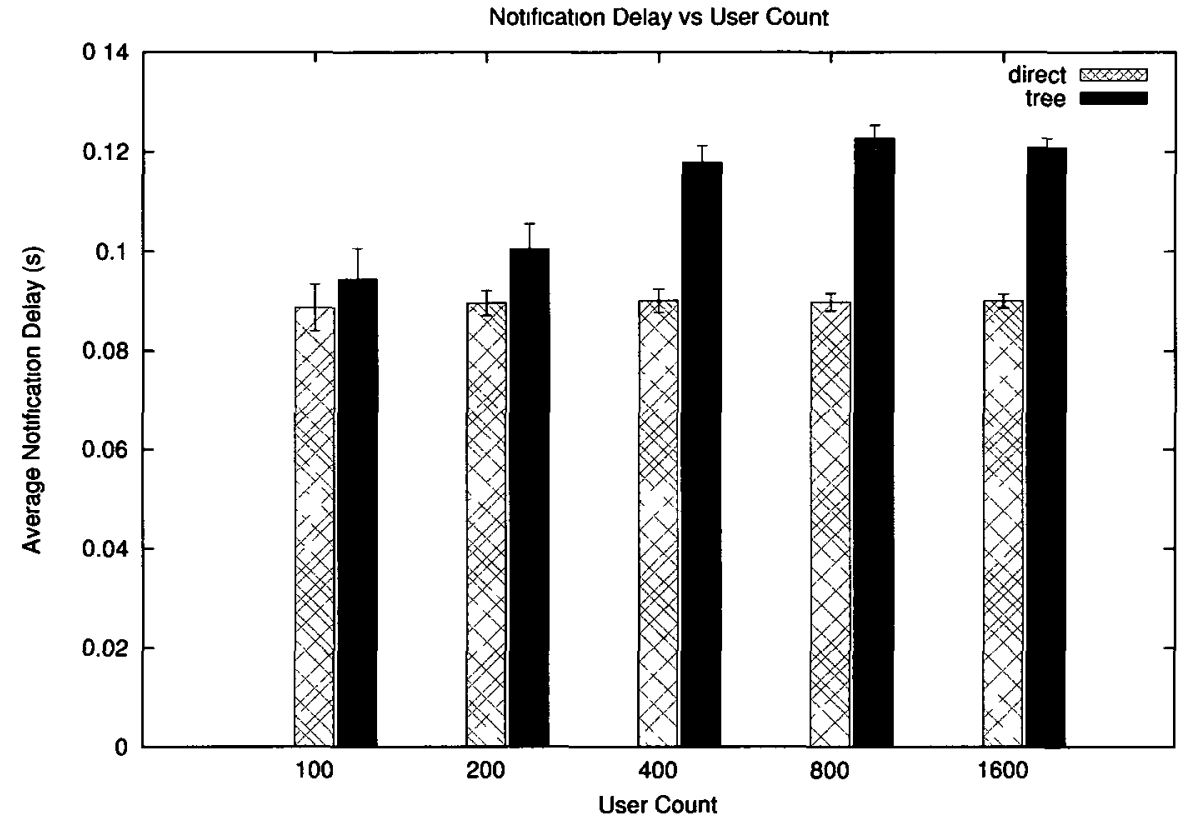

Figure 5.11: average notification delay

\subsubsection{Scalability}

The scalability of tree-based notifications requires evaluating the results of using the protocol as the number of users of the service increase. Figure 5.11 and 5.12 are the results of varying the number of users, which were assigned to a fixed set of 100 RLS nodes, with no node failure or dropped packets.

The results show that traffic cost is almost cut in half for tree-based notifications unless there are less than 400 simulated users. Tree-based notifications also have a small additional notification delay which seems to stop increasing as more than 400 users are used. This fixed ratio for traffic cost and near constant average for average notification delay appears to show a limit on the scalability of the protocol when the 


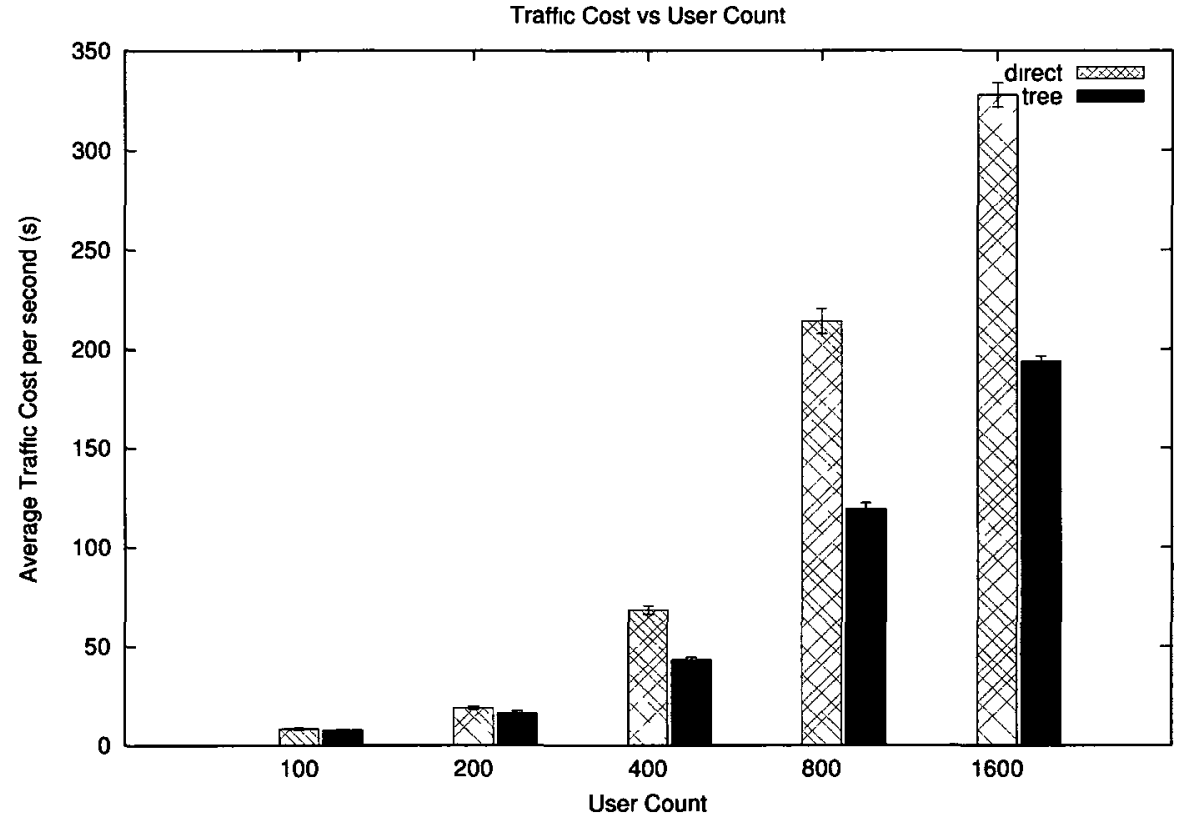

Figure 5.12: average traffic cost

number of RLS nodes are fixed.

These results show that the protocol is able to handle an increase in users quite well even without adding additional presence server nodes. 


\section{Chapter 6}

\section{Conclusions}

The simulation results show improvement on standard IMS presence. The traffic cost was reduced without significantly affecting the average delay. We attribute this to our low overhead tree building protocol that uses NCs to place watchers in notification trees. The lack of overhead makes our protocol useful even for transferring relatively small quantities of data. Further work aims to improve these results.

IMS presence traffic was simulated using some real world data. The similarity between social networks and presence relationships were used to model the behavior of users, and ping traces were used to simulate network delay. There are still limitations for the simulation to address.

Our proposed SIP extensions apply to the SIP event notification framework in general such that it could directly be used for other SIP event packages besides presence. Therefore, the benefits of using NCs for tree-based notification dissemination are worth pursuing further. The heuristics that are used for building a notification tree could also be used for other systems that do not use SIP. 


\subsection{Summary of Contributions}

1. Developed a low-overhead tree-building distribution system for notifications that significantly reduces network traffic compared to direct notifications, adds only a minor notification delay, reliably handles network faults, and increases scalability of the presence service.

2. Provided a compatible way of extending the SIP event notification framework for tree-based event notification. The protocol extension can be used between nodes that advertise support for it, but will use direct notifications where one party does not advertise support for the extension.

3. Evaluated the performance of tree-based notification for the SIP event notification framework using a simulator. The performance was evaluated in the presence of network faults. These faults include server crashes and packet loss.

\subsection{Future Work}

The presented research could be extended in the future in the following ways:

1. Possible improvements to the tree building protocol, proposed in chapter 4 , could be evaluated. In particular, equation (4.1) could be used to replace nonroot parent nodes with a new child if this could lower the family delay.

2. Compatibility with IP Multicast could be integrated into the solution using the approach taken for Island Multicast [19], but using the network coordinate based hierarchical notification distribution proposed in chapter 4 . 
3. Implementing the protocol extension could be done in order to test the protocol outside of the simulator. These tests could use actual users in order to provide realistic traffic behaviour, and would allow for acceptance testing to be done on the resulting user experience. The implementation could be used as a reference for proposing an extension to the Presence Event Package. 


\section{List of References}

[1] S. Islam and J. W. Atwood, "Sender access control in IP multicast," in 32nd IEEE Conference on Local Computer Networks, (Dublin, Ireland), pp. 79-86, 2007.

[2] D. A. H. Smith, C. Huang, and J. Yan, "Hierarchical notification dissemination for IMS presence using network coordinates," in Proceedings of the 2010 IEEE Global Communicatıons Conference, (Miami, FL, USA), 2010.

[3] J. Rosenberg, "A presence event package for the session initiation protocol (SIP)." RFC 3856, August 2004.

[4] R. B. Jennings, E. M. Nahum, D. P. Olshefski, D. Saha, Z.-Y. Shae, and C. Waters, "A study of internet instant messaging and chat protocols," IEEE Network, vol. 20 , no. 4 , pp. 16-21, 2006.

[5] G. Camarillo and M. A. Garcia-Martin, The 3G IP multımedıa subsystem (IMS): merging the Internet and the cellular worlds. Wiley, 2004.

[6] A. B. Roach, "Session initiation protocol (SIP)-specific event notification." RFC 3265, June 2002.

[7] A. B. Roach, B. Campbell, and J. Rosenberg, "A session initiation protocol (SIP) event notification extension for resource lists." RFC 4662, August 2006.

[8] P. Mockapetris, "Domain names - implementation and specification." RFC 1035, November 1987.

[9] A. Niemi, "Session initiation protocol (SIP) extension for event state publication." RFC 3903, October 2004. 
[10] J. Rosenberg, H. Schulzrinne, G. Camarillo, A. Johnston, J. Peterson, R. Sparks, M. Handley, and E. Schooler, "SIP: Session initiation protocol." RFC 3261, June 2002.

[11] H. Sugano, S. Fujimoto, G. Klyne, A. Bateman, W. Carr, and J. Peterson, "Presence information data format (PIDF)." RFC 3863, August 2004.

[12] H. Schulzrinne, V. Gurbani, P. Kyzivat, and J. Rosenberg, "RPID: Rich presence extensions to the presence information data format (PIDF)." RFC 4480, July 2006 .

[13] H. Schulzrinne, "CIPID: Contact information for the presence information data format." RFC 4482, July 2006.

[14] H. Schulzrinne, "Timed presence extensions to the presence information data format (PIDF) to indicate status information for past and future time intervals." RFC 4481, July 2006.

[15] M. Lonnfors and K. Kiss, "Session initiation protocol (SIP) user agent capability extension to presence information data format (PIDF)." RFC 5196, September 2008.

[16] F. Dabek, R. Cox, F. Kaashoek, and R. Morris, "Vivaldi: a decentralized network coordinate system," in Proceedings of the 2004 conference on Applucatıons, technologies, architectures, and protocols for computer communıcatıons, (New York, NY, USA), pp. 15-26, ACM, 2004.

[17] Y. Chen, Y. Xiong, X. Shi, J. Zhu, B. Deng, and X. Li, "Pharos: accurate and decentralised network coordinate system," IET Communications, vol. 3, no. 4, pp. 539-548, 2009.

[18] A. Ikram, M. Zafar, N. Baker, and R. Chiang, "IMS-MBMS convergence for next generation mobile networks," in The 2007 International Conference on Next Generation Mobule Applıcatıons, Services and Technologies, (Washington, DC, USA), pp. 49-56, IEEE Computer Society, 2007. 
[19] K.-W. R. Cheuk, S.-H. G. Chan, and J. Y.-B. Lee, "Island multicast: The combination of ip multicast with application-level multicast," in 2004 IEEE Internatıonal Conference on Communıcatıons, vol. 3, (Paris, France), pp. 1441-1445, 2004 .

[20] B. Zhang, W. Wang, S. Jamin, D. Massey, and L. Zhang, "Universal IP multicast delivery," Computer Networks: The international Journal of Computer and Telecommunications Networking, vol. 50, pp. 781-806, 2006.

[21] A. Kanamaru, Y. Tsuchiya, T. Fujisaki, and K. Takahashi, "Fieldcast2: Flexible P2P architecture for presence information sharing," in Proceedings of the 2nd IEEE Annual Conference on Pervasive Computıng and Communications Workshops, (Orlando, FL, USA), pp. 98-103, IEEE, 2004.

[22] J. Ledlie, P. Gardner, and M. I. Seltzer, "Network coordinates in the wild," in Network Systems Design and Implementation, (Cambridge, MA, USA), USENIX, 2007.

[23] R. Lin, H. Zou, Y. Zhao, and F. Yang, "A novel approach to optimize information dissemination in IMS presence system," in Next Generation Teletraffic and Wired/Wireless Advanced Networking, vol. 5174 of Lection Notes in Computer Scrence, pp. 187-198, Springer-Verlag, 2008.

[24] K. Chen and K. Nahrstedt, "Effective location-guided tree construction algorithms for small group multicast in MANET," in INFOCOM 2002. The 21st Annual Joint Conference of the IEEE Computer and Communications Societıes, vol. 3, (New York, NY, USA), pp. 1180-1189, 2002.

[25] P. Pietzuch, J. Ledlie, and M. Seltzer, "Supporting network coordinates on PlanetLab," in Proceedings of the 2nd conference on Real, Large Distributed Systems, (Berkeley, CA, USA), pp. 19-24, USENIX Association, 2005.

[26] A. Mislove, M. Marcon, K. P. Gummadi, P. Druschel, and B. Bhattacharjee, "Measurement and analysis of online social networks," in Proceedings of the 7th ACM SIGCOMM conference on Internet measurement, (New York, NY, USA), pp. 29-42, ACM, 2007. 
[27] A. Harrowell, "A pointless multimedia subsystem?," Mobıle Communications International, vol. 135, pp. 6-7, October 2006.

[28] P. L'Ecuyer, "Good parameters and implementations for combined multiple recursive random number generators," Operations Research, vol. 47, pp. 159-164, 1999.

[29] K. Fall and K. Varadhan, "The ns Manual (formerly ns Notes and Documentation)," The VINT project, 2010. 


\title{
Appendix A
}

\section{Presence Information Data Elements}

\section{A.1 Presence Information Data Format (PIDF)}

\author{
$[11]$ \\ $<$ presence $>$ The root XML element. \\ $<$ tuple> Container element that is used to group presence data from a specific \\ device or application. \\ <status > Specifies whether the presentity is online or offline (mandatory). \\ $<$ contact $>$ URI at which to contact the presentity (e.g. telephone number or SIP \\ address). \\ $<$ note $>$ Human readable note left by the presentity. \\ $<$ timestamp $>$ The time the presence status was last changed.
}




\section{A.2 Rich Presence Information Data Format (RPID) [12]}

$<$ activity $>$ What the presentity is currently doing (e.g. on the phone).

$<$ class $>$ Allows tuples to be grouped by class.

$<$ idle $>$ The last time the device was used.

$<$ place-type $>$ Indicates the type of place in which the presentity is located (e.g. home, office, library, theatre, hotel restaurant).

$<$ deviceID $>$ A reference to the device associated with the tuple.

$<\operatorname{mood}>$ Indicates the mood of the presentity.

$<$ place-is $>$ Reports on the properties of the place (e.g. dark, noisy).

$<$ privacy $>$ The degree of privacy the presentity currently has for a conversation by indicating whether others may observer the communication.

$<$ relationship $>$ The relationship the presentity has with another contact (e.g. family, assistant, supervisor).

$<$ sphere $>$ The current role of the presentity (e.g. home, work).

$<$ status-icon $>$ A custom status icon depicting the current status of the presentity.

$<$ time-offset $>$ The presentity's current time zone. 
<user-input > The usage state of different human user input (e.g. keyboard, mouse, voice) that may indicate the last input time or simply that the user-input is active or idle.

\section{A.3 Contact Information in Presence Information Data Format (CIPID) [13]}

$<$ card > URL pointing to a digital business card.

$<$ display-name $>$ The name the presentity suggests to be shown to watchers.

$<$ homepage $>$ URL of the presentity's home page.

$<$ icon $>$ URL pointing to an image that represents the presentity for watchers (e.g. a picture of the presentity).

$<$ sound $>$ A sound that the presentity has suggested to be played for watchers when the presentity's status changes to online.

\section{A.4 Timed Presence Extension [14]}

<time-status $>$ Indicates the status of the presentity between a range of time either in the past, future, or present (e.g. to indicate that the presentity is offline during a meeting or vacation). 


\section{A.5 User Agent Capability Extension [15]}

$<$ servcaps $>$ The root element for service capabilities. This contains all the following elements of this extension, with exception for devcaps and mobility.

$<$ audio $>$ A boolean value that indicates support for audio as a streaming media type.

$<$ application $>$ A boolean value that indicates support for application as a streaming media type.

$<$ data $>$ A boolean value that indicates support for data as a streaming media type.

$<$ control $>$ A boolean value that indicates support for control as a streaming media type.

$<$ video $>$ A boolean value that indicates support for video as a streaming media type.

$<$ text $>$ A boolean value that indicates support for text as a streaming media type.

$<$ message $>$ A boolean value that indicates support for messaging as a streaming media type.

$<$ type $>$ MIME content type that is supported by the service.

<automata $>$ A boolean value that indicates whether it is an automated service (e.g. voicemail).

$<$ class $>$ Indicate whether the service may or may not be used for business or personal communication. 
$<$ duplex $>$ States whether the communication service may simultaneously send and receive, alternate between the two, just send, or just receive.

$<$ description $>$ A textual description of the device or service

$<$ event-packages $>$ A list of supported event packages.

$<$ priority $>$ Contains a list of supported and unsupported ranges of call priorities.

$<$ methods $>$ Lists the sip methods supported or not supported by the service.

<extensions $>$ Lists SIP extensions that are supported or not supported by the service.

<schemes > List of supported or unsupported URI schemes.

<actor > Indicates whether the service provides communication with the principal directly, through an intermediary to contact the principal, through a proxy, or only provides information about the principal.

<isfocus $>$ A boolean value indicating support for conferencing.

$<$ languages $>$ A list of languages supported by the service.

$<$ devcaps $>$ The root element for device capabilities. This includes the mobility and description elements.

$<$ mobility $>$ Indicates whether the device supports a mobile or fixed connection network connection. 